\title{
THE THRESHOLD THEOREM
}

\section{FOR THE $(4+1)$-DIMENSIONAL YANG-MILLS EQUATION: AN OVERVIEW OF THE PROOF}

\author{
SUNG-JIN OH AND DANIEL TATARU
}

\begin{abstract}
This article is devoted to the energy critical hyperbolic Yang-Mills equation in the $(4+1)$-dimensional Minkowski space, which is considered by the authors in a sequence of four papers. The final outcome of these papers is twofold: (i) the Threshold Theorem, which asserts that global well-posedness and scattering hold for all topologically trivial initial data with energy below twice the ground state energy; and (ii) the Dichotomy Theorem, which for larger data in arbitrary topological classes provides a choice of two outcomes, either a global scattering solution or a soliton bubbling off. In the last case, the bubbling-off phenomena can happen in one of two ways: (a) in finite time, triggering a finite time blowup; or (b) in infinite time. Our goal here is to first describe the equation and the results, and then to provide an overview of the flow of ideas within their proofs in the above-mentioned four papers.
\end{abstract}

\section{CONTENTS}

1. Introduction

2. The caloric gauge

3. Energy dispersed caloric Yang-Mills waves 192

4. Large data, causality, and the temporal gauge 197

5. To bubble or not to bubble 201

\begin{tabular}{l|l|}
\hline About the authors & 208 \\
\hline
\end{tabular}

References

\section{INTRODUCTION}

The purpose of this expository article is to provide an overview of the authors' recent series of work [30 33, in which a positive answer to the Threshold Conjecture for the energy critical hyperbolic Yang-Mills equation is given.

Received by the editors May 22, 2018.

2010 Mathematics Subject Classification. Primary 35L70, 70S15.

The first author was supported by the Miller Research Fellowship from the Miller Institute, UC Berkeley, and the TJ Park Science Fellowship from the POSCO TJ Park Foundation.

The second author was partially supported by the NSF grant DMS-1266182 as well as by a Simons Investigator grant from the Simons Foundation.

Part of the work described here was carried out during the semester-long program "New Challenges in PDE" held at MSRI in the fall of 2015. 
1.1. Yang-Mills as a generalization of Maxwell. A natural way to introduce and motivate our subject - the hyperbolic Yang-Mills equation - is to view it as a geometric generalization of the (sourceless) Maxwell equation, the basic equation in electromagnetism.

In its most elementary form, the Maxwell equation for the time-dependent vector fields $(\mathbf{E}(t), \mathbf{B}(t))$ (called the electric and magnetic fields, respectively) on $\mathbb{R}^{3}$ reads

$$
\left\{\begin{array}{l}
\partial_{t} \mathbf{E}=\nabla \times \mathbf{B}, \quad \partial_{t} \mathbf{B}=-\nabla \times \mathbf{E}, \\
\nabla \cdot \mathbf{E}=0, \quad \nabla \cdot \mathbf{B}=0
\end{array}\right.
$$

To describe its generalization to the hyperbolic Yang-Mills equation, it is helpful to reformulate (1.1) in three successive steps.

(1) Covariant formulation in $\mathbb{R}^{1+d}$. The natural geometric setting for the Maxwell equation is the Minkowski spacetime $\left(\mathbb{R}^{1+3}, \mathbf{m}\right)$, which is equipped with the Minkowski metric $\mathbf{m}=\operatorname{diag}(-1,+1,+1,+1)$ in the rectilinear coordinates $\left(t=x^{0}, x^{1}, x^{2}, x^{3}\right)$. The relativistic invariance of the Maxwell equation (i.e., invariance with respect to the isometries of $\left.\left(\mathbb{R}^{1+3}, \mathbf{m}\right)\right)$ is most manifest if we combine $\mathbf{E}, \mathbf{B}$ and introduce the electromagnetic field $F$, which is a 2 -form on $\mathbb{R}^{1+3}$ related to $\mathbf{E}, \mathbf{B}$ by

$$
\mathbf{E}_{i}=F_{0 i}, \quad \mathbf{B}_{i}=-\frac{1}{2} \sum_{j, k=1}^{3} \epsilon_{i j k} F_{j k} .
$$

Then the system (1.1) can be conveniently rewritten in the form

$$
\begin{aligned}
\partial^{\mu} F_{\nu \mu} & =0, \\
d F & =0,
\end{aligned}
$$

where we use the standard index convention for tensors (by summing up repeated upper and lower indices and by raising/lowering indices using $\mathbf{m}$ ), and $d$ is the exterior derivative.

In contrast to (1.1), the covariant formulation (1.2)-(1.3) easily lends itself to generalization to other dimensions $\mathbb{R}^{1+d}$. In what follows, we will work under this generality.

(2) Action principle formulation. In order to give an action principle (i.e., a Lagrangian field theory description) of the Maxwell equation, we introduce an electromagnetic potential $A$, which is a real-valued 1 -form on $\mathbb{R}^{1+d}$ related to $F$ by

$$
F=F[A]=d A .
$$

Note that the existence of such a 1 -form $A$ is equivalent to (1.3), as $\mathbb{R}^{1+d}$ is contractible. Moreover, (1.2) is the Euler-Lagrange equation for the forma 1 action

$$
\mathcal{L}(A)=\int_{\mathbb{R}^{1+d}} F[A]^{\mu \nu} F[A]_{\mu \nu} d x^{0} \cdots d x^{d}
$$

\footnotetext{
${ }^{1}$ The integral (1.4) may not be well-defined as there is no a priori reason for $F[A]$ to decay. However, the variation of $\mathcal{L}(A)$ under compactly supported variations $A \mapsto A+\delta A, \delta A \in C_{0}^{\infty}\left(\mathbb{R}^{1+d}\right)$ is defined. Formal critical points and the Euler-Lagrange equation for $\mathcal{L}(A)$ are formulated with respect to such variations.
} 
(3) Gauge-theoretic formulation. Observe that the description of $F$ in terms of $A$ suffers from the following ambiguity: for any real-valued function $\chi$ on $\mathbb{R}^{1+d}$, we have

$$
F[A]=d A=d(A-d \chi)=F[A-d \chi] .
$$

This property, called gauge invariance, admits a particularly natural interpretation if we take the following gauge-theoretic viewpoint: Consider a vector bundle $E$ over $\mathbb{R}^{1+d}$ with structure group $U(1)$, and view $i A$ as a (global) connection 1-form associated with a connection $\mathbf{D}$ on $E$ (i.e., $\mathbf{D}=d+i A$ in a global trivialization of the bundle, which exists since the base space $\mathbb{R}^{1+d}$ is contractible), and $i F=i d A$ as the curvature 2 -form associated to $\mathbf{D}$. Then $A \mapsto A-d \chi$ is nothing but the transformation law for $A$ under the fiberwise multiplication by $e^{i \chi}$ (which is called a gauge transformation), and (1.5) is the standard covariance property of the curvature 2-form.

In short, the hyperbolic Yang-Mills equation is a generalization of the gaugetheoretic formulation of the Maxwell equation, where the group $U(1)$ is replaced by an arbitrary compact Lie group $\mathbf{G}$.

To be precise, we need to introduce some notation. Let $\mathbf{D}$ be a connection on a vector bundle with structure group G. In a global trivialization we may write

$$
\mathbf{D}=d+A,
$$

where $A$ is a 1 -form taking values in the Lie algebra $\mathfrak{g}$ of $\mathbf{G}$, and is called a connection 1 -form associated with $\mathbf{D}$. In what follows, we will simply identify $\mathbf{D}$ with $A$. The commutator $\mathbf{D}_{\mu} \mathbf{D}_{\nu}-\mathbf{D}_{\nu} \mathbf{D}_{\mu}$ gives rise to the curvature 2 -form

$$
F_{\mu \nu}=F[A]_{\mu \nu}=\partial_{\mu} A_{\nu}-\partial_{\nu} A_{\mu}+\left[A_{\mu}, A_{\nu}\right] .
$$

Under the fiberwise multiplication by $O(t, x) \in \mathbf{G}$ (such a function $O: \mathbb{R}^{1+d} \rightarrow \mathbf{G}$ is called a gauge transformation), $A$ and $F$ transform in the following fashion,

$$
A \mapsto \mathcal{G}(O) A_{\mu}=\operatorname{Ad}(O) A_{\mu}-O_{; \mu}, \quad F \mapsto \operatorname{Ad}(O) F,
$$

where $O_{; \mu}=O^{-1} \partial_{\mu} O$, and $\operatorname{Ad}(\cdot)(\operatorname{resp} \cdot \operatorname{ad}(\cdot))$ is the adjoint action of $\mathbf{G}(\operatorname{resp} . \mathfrak{g})$ on $\mathfrak{g}$. If $\mathbf{G}$ is a matrix group, then

$$
\operatorname{Ad}(O) A=O A O^{-1}, \quad \operatorname{ad}(A) B=[A, B], \quad(O \in \mathbf{G}, A, B \in \mathfrak{g}) .
$$

We say that $A$ solves the hyperbolic Yang-Mills equation if it is a formal critical point for the Yang-Mills action

$$
\mathcal{L}(A)=\int_{\mathbb{R}^{1=d}}\left\langle F[A]^{\mu \nu}, F[A]_{\mu \nu}\right\rangle d \mathrm{Vol},
$$

where $\langle\cdot, \cdot\rangle$ is an inner product on $\mathfrak{g}$, which is bi-invariant in the sense that

$$
\langle\operatorname{Ad}(O) A, \operatorname{Ad}(O) B\rangle=\langle A, B\rangle \quad \text { for all } O \in \mathbf{G}, \quad A, B \in \mathfrak{g} .
$$

The Euler-Lagrange equation, which will be called the hyperbolic Yang-Mills equation, takes the form

$$
\mathbf{D}^{\mu} F_{\nu \mu}=0,
$$

where $\mathbf{D}_{\mu}$ acts on $F$ by the formula $\mathbf{D}_{\mu} F_{\alpha \beta}=\left(\partial_{\mu}+\operatorname{ad}\left(A_{\mu}\right)\right) F_{\alpha \beta}$. 
The Yang-Mills action (1.9) and the hyperbolic Yang-Mills equation (1.10) are gauge invariant, i.e., they remain unchanged under (1.8). Moreover, they are invariant under the natural scaling

$$
A \mapsto A_{(\lambda)}(t, x):=\lambda A(\lambda t, \lambda x) .
$$

Note that (1.7) and (1.10) are nonlinear if $\mathbf{G}$ is noncommutative, i.e., if the Lie bracket is nontrivial on $\mathfrak{g}$. Conversely, (1.7) and (1.10) reduces to the Maxwell equation when $\mathbf{G}$ is commutative, e.g., $\mathbf{G}=U(1)$.

\subsection{IVP for Yang-Mills and the threshold conjecture in the energy crit-} ical case. Like the Maxwell equation, it is natural to pose the initial value problem (IVP) for the hyperbolic Yang-Mills equation, which is formulated in a gauge invariant manner as follows. Given a pair $(a, e)$ of $\mathfrak{g}$-valued 1 -forms, we say that $A$ solves the IVP with the initial condition $(a, e)$ if $A$ satisfies (1.10) and

$$
\left(A_{j}, F_{0 j}\right)(t=0)=\left(a_{j}, e_{j}\right) .
$$

Note that the $\nu=0$ component of (1.10) reduces to $\mathbf{D}^{\ell} F_{0 \ell}=0$, which involves no second-order derivatives in time. Hence, any initial data $(a, e)$ for (1.12) must obey the constraint equation

$$
\mathbf{D}^{\ell} e_{\ell}=0
$$

where $\mathbf{D}_{j}=\partial_{j}+\operatorname{ad}\left(a_{j}\right)$; this is precisely the analogue of the Gauss equation $\nabla \cdot \mathbf{E}=0$ in electromagnetism. Conversely, it is a standard result that for any smooth initial data $(a, e)$ satisfying (1.13), there exists a local solution to the initial value problem, which is unique up to gauge invariance.

In order to understand the global behavior of solutions, a fundamental role is played by the conserved energy

$$
\mathcal{E}_{\{t\} \times \mathbb{R}^{d}}(A)=\frac{1}{2} \int_{\{t\} \times \mathbb{R}^{d}} \sum_{0 \leq \mu<\nu \leq d}\left|F_{\mu \nu}\right|^{2} d x,
$$

which is constant in $t$ for any solution $A$. Depending on the underlying (spatial) dimension $d$, the conserved energy behaves differently under the natural scaling $A \mapsto A_{(\lambda)}(t, x):=\lambda A(\lambda t, \lambda x)$ of (1.10). A distinguished dimension is the energy critical case $d=4$, in which we have the invariance

$$
\mathcal{E}_{\{t\} \times \mathbb{R}^{4}}(A)=\mathcal{E}_{\left\{\lambda^{-1} t\right\} \times \mathbb{R}^{4}}\left(A_{(\lambda)}\right) .
$$

This is a particularly interesting borderline case: on the one hand, the conserved energy is weak enough to allow for singularity formation (see, for instance, [15, 34]), but on the other hand it is strong enough to allow for nontrivial characterization of such phenomena (as exemplified by our results in section 1.7 below). In the remainder of this article, we will focus exclusively on the energy critical case, i.e., the hyperbolic Yang-Mills equation in $\mathbb{R}^{1+4}$.

Recently, the second author with J. Krieger proved the following definitive result in the small energy case:

Theorem 1.1 ([20]). The IVP for the hyperbolic Yang-Mills equation in $\mathbb{R}^{1+4}$ is globally well-posed, and the solutions scatter (in a suitable gauge) for all initial data with sufficiently small energy. 
We remark that the notions of well-posedness and scattering for the hyperbolic Yang-Mills equation must be formulated carefully, keeping the gauge invariance property in mind; we will return to this issue in section 1.6.

Theorem 1.1 naturally raises the following question: What is the sharp energy threshold, below which global well-posedness and scattering hold? A clue for the answer is provided by the existence of a ground state $Q$, which is the least energy nontrivial steady state (i.e., time independent solution) for the hyperbolic YangMills equation; see section [1.5. The ground state represents the exact balance between the stabilizing linear part and the destabilizing nonlinearity, and as such is a natural candidate for the threshold between global well-posedness/scattering vs. blowup. This discussion motivates the following:

Threshold Conjecture. The IVP for the hyperbolic Yang-Mills equation in $\mathbb{R}^{1+4}$ is globally well-posed and the solutions scatter (in a suitable gauge) for all initial data with energy below that of the ground state.

Our recent series of work 30 33. provides a positive answer to (a sharper version of) the Threshold Conjecture and, moreover, provides a description of possible failure of global well-posedness/scattering in general. Before describing the main results, however, let us make a slight detour and discuss the broader context in which the above conjecture and our results fit in most naturally.

1.3. Energy critical geometric wave equations and the elliptic/parabolic counterparts. The $(4+1)$-dimensional hyperbolic Yang-Mills equation is a prime example of a distinguished class of nonlinear wave equations, which we will call the energy critical geometric wave equations. These equations arise naturally in connection with physics and geometry (see Table 1 for the latter point) and have been studied extensively in recent years.

- Energy critical nonlinear wave $(N L W)$. The simplest example in this class is the following PDE for a real-valued function $u: \mathbb{R}^{1+d} \rightarrow \mathbb{R}$ with a pure power-type nonlinearity:

$$
\square u=\varepsilon|u|^{\frac{4}{d-2}} u .
$$

Here, $\square=\partial^{\mu} \partial_{\mu}=-\partial_{t}^{2}+\Delta$ is the usual (scalar) d'Alembertian and $\varepsilon \in\{+1,-1\}$; (NLW) is said to be defocusing (resp. focusing) if $\varepsilon=-1$ (resp. $\varepsilon=+1$ ). The power $\frac{4}{d-2}$ is chosen precisely so that the conserved energy

$$
\mathcal{E}_{\{t\} \times \mathbb{R}^{d}}(u)=\frac{1}{2} \int_{\{t\} \times \mathbb{R}^{d}} \sum_{\mu=0}^{d}\left|\partial_{\mu} u\right|^{2}+\varepsilon \frac{d-2}{d}|u|^{\frac{2 d}{d-2}} d x
$$

is critical (i.e., invariant) with respect to the invariant scaling $u \mapsto \lambda^{\frac{d-2}{2}} u(\lambda t, \lambda x)$ for (NLW).

- Wave maps $(W M)$. The wave maps are generalizations of solutions to the scalar wave equation $\square u=0$ to manifold-valued maps. In the action principle formulation, a wave map is a (formal) critical point of the action

$$
\mathcal{L}(\Phi)=\frac{1}{2} \int_{\mathbb{R}^{1+d}}\left\langle\partial_{\mu} \Phi, \partial^{\mu} \Phi\right\rangle_{\mathbf{h}} d \mathrm{Vol}
$$

where $(\mathcal{N}, \mathbf{h})$ is a Riemannian manifold and $\Phi$ is a map $\mathbb{R}^{1+d} \rightarrow \mathcal{N}$. The corresponding Euler-Lagrange equation is called the wave maps equation. When $\mathcal{N}$ 
is isometrically embedded in some Euclidean space $\left(\mathbb{R}^{N}, \delta\right)$, it takes the form $(\mathrm{WM})$

$$
\square \Phi=-\mathcal{A}(\Phi)\left(\partial^{\mu} \Phi, \partial_{\mu} \Phi\right)
$$

where $\mathcal{A}$ is the second fundamental form of the embedding $(\mathcal{N}, \mathbf{h}) \hookrightarrow\left(\mathbb{R}^{N}, \delta\right)$. (WM) has a nonlinearity originating from the curvature of $\mathcal{N}$, and indeed it reduces to the linear scalar wave equation when $(\mathcal{N}, \mathbf{h})=\left(\mathbb{R}, d x^{2}\right)$.

The conserved energy for (WM) is

$$
\mathcal{E}_{\{t\} \times \mathbb{R}^{d}}(\Phi)=\frac{1}{2} \int_{\{t\} \times \mathbb{R}^{d}} \sum_{\mu=0}^{d}\left|\partial_{\mu} \Phi\right|_{\mathbf{h}}^{2} d x
$$

and the invariant scaling is $\Phi \mapsto \Phi(\lambda t, \lambda x)$. After a simple computation, we see that (WM) is energy critical in the case $d=2$.

- Maxwell-Klein-Gordon (MKG) equation. As the name suggests, the MaxwellKlein-Gordon equation is coupled system of a Klein-Gordon field $\phi$, viewed as a section of a $U(1)$-bundle, and a Maxwell connection $\mathbf{D}=d+i A$ :

$(\mathrm{MKG})$

$$
\left\{\begin{aligned}
\partial^{\mu} F_{\nu \mu} & =\operatorname{Im}\left(\phi \overline{\mathbf{D}_{\nu} \phi}\right) \\
\mathbf{D}^{\mu} \mathbf{D}_{\mu} \phi & =m^{2} \phi
\end{aligned}\right.
$$

This system, especially in the massless case $m=0$, is often studied as a simpler nonlinear model for the Yang-Mills equation (in that its structure group is still the commutative group $U(1))$.

When $m=0$, the conserved energy for (MKG) is

$$
\mathcal{E}_{\{t\} \times \mathbb{R}^{d}}(A, \phi)=\frac{1}{2} \int_{\{t\} \times \mathbb{R}^{d}} \sum_{0 \leq \mu<\nu \leq d}\left|F_{\mu \nu}\right|^{2}+\sum_{\mu=0}^{d}\left|\mathbf{D}_{\mu} \phi\right|^{2} d x
$$

and the invariant scaling is $(A, \phi) \mapsto \lambda(A, \phi)(\lambda t, \lambda x)$. Exactly as in the YangMills case, we see that (MKG) is energy critical when $d=4$.

As a culmination of the tremendous activity in the past few decades, the Threshold Conjecture was resolved for all of the above equations; see 6, 7, 38, for the defocusing NLW, [10] for the focusing NLW, 17, 40, 41, 46, 50] for the energy critical WM, and finally [16, 27 29] for the energy critical, massless MKG equation 2 As it will be pointed out several times in this expository article, our present work on the hyperbolic Yang-Mills equation builds upon this large body of work.

Like the Yang-Mills equation, (NLW) and (WM) are the hyperbolic (or wave) counterparts of celebrated elliptic and parabolic equations in differential geometry. The elliptic equations arise as the time-independent restrictions of the above hyperbolic equations, and the parabolic equations arise as the gradient flows for the time-independent actions. These correspondences are summarized in Table ${ }^{3}{ }^{3}$

In the context of these analogies, the small energy result (Theorem 1.1) corresponds to the classical $\epsilon$-regularity theorems in the elliptic and parabolic cases, and the Threshold Conjecture is naturally understood as a part of the well-known bubbling phenomenon for the elliptic and parabolic equations in the conformally

\footnotetext{
${ }^{2}$ We note, however, that the large data problem for the energy critical, massive (i.e., $m \neq 0$ ) Maxwell-Klein-Gordon equation is still open; see the recent work [5] for the small energy case.

${ }^{3}$ Another excellent example of such an analogy would be the Einstein equation/the (Riemannian) Einstein metric condition/Ricci flow. However, due to their severe nonlinearity as well as their richer geometric properties, the general discussion in this subsection does not seem to apply directly to these equations.
} 
TABLE 1. Elliptic/parabolic counterparts for geometric wave equations

\begin{tabular}{|c|c|c|}
\hline Hyperbolic & Elliptic & Parabolic \\
\hline $\begin{array}{c}\text { energy critical NLW } \\
\text { wave maps }\end{array}$ & $\begin{array}{l}\text { Yamabe equation } \\
\text { harmonic maps }\end{array}$ & $\begin{array}{c}\text { Yamabe flow } \\
\text { harmonic map heat flow }\end{array}$ \\
\hline hyperbolic Yang-Mills & harmonic Yang-Mills & Yang-Mills heat flow \\
\hline
\end{tabular}

invariant case; see, for instance, the monographs 4, 9, 22, 43, and the references therein. Although the analytic issues and techniques differ considerably (as is evident in the overview of our proof below), analogies with classical elliptic/parabolic cases have provided a powerful guiding principle for the hyperbolic problem at hand 4

An interesting aspect of our work on the Threshold Conjecture for the hyperbolic Yang-Mills equation is that not only does the elliptic counterpart (namely, the harmonic Yang-Mills equation) make a direct appearance in the proof, but so does the parabolic counterpart (namely, the Yang-Mills heat flow); see section 1.6.

1.4. Topological classes of finite energy connections on $\mathbb{R}^{4}$. The goal of the remainder of the Introduction is to present the main results of the authors' recent series of work [30 33, which in particular gives an affirmative answer to the Threshold Conjecture; see section 1.7. In this subsection, we describe the topological structure of the space of finite energy connections in $\mathbb{R}^{4}$, which is closely tied to the theory of the harmonic Yang-Mills equation (see section 1.5) and which is also important for formulating the sharp version of the Threshold Conjecture (see Theorem 1.4).

The space of finite energy connections in $\mathbb{R}^{4}$ is not connected. Instead, such connections can be classified in terms of their topological class; see section 4 for more details. For a compact base manifold, such as $\mathbb{S}^{4}$, this term refers to the isomorphism classes of principal G-bundles which support the connection. On the other hand, for $\mathbb{R}^{4}$, which is contractible and thus supports only the trivial fiber bundles, a topological class must be interpreted rather as a property of a connection.

In the particular case of four-dimensional $\mathrm{SU}(2)$ connections, the topological class is easily described in terms of the (second) Chern number

$$
c_{2}=\frac{1}{8 \pi^{2}} \int_{\mathbb{R}^{4}} \operatorname{tr}(F \wedge F) .
$$

This is always an integer if $A$ has finite energy. For an arbitrary compact noncommutative Lie group, we have an analogue of $c_{2}$,

$$
\chi(A)=\int_{\mathbb{R}^{4}}-\langle F \wedge F\rangle=\frac{1}{4} \int_{\mathbb{R}^{4}}-\left\langle F_{i j}, F_{k \ell}\right\rangle d x^{i} \wedge d x^{j} \wedge d x^{k} \wedge d x^{\ell},
$$

which we denote by $\chi(A)$ and call the characteristic number. This quantity is still a topological invariant, but it no longer fully describes the topological class.

The connections which are in the same class as the zero connection are called topologically trivial. For such connections, $\chi=0$. An alternative way to describe topologically trivial connections is given by the following result, which generalizes Uhlenbeck's lemma [53]:

\footnotetext{
${ }^{4}$ Readers familiar with the bubbling phenomenon in the elliptic/parabolic cases are invited to compare it with our main theorems in section 1.7
} 
Theorem 1.2 ([32]). A finite energy connection $A$ in $\mathbb{R}^{4}$ is topologically trivial if and only if $A \in \dot{H}^{1}$ in a suitable gauge.

Since scattering implies asymptotic convergence to the zero connection in a certain sense, one would expect that, under any reasonable formulation, any scattering solution to the hyperbolic Yang-Mills connection must be topologically trivial at each $t$. Therefore, the class of topologically trivial connections is the most natural setting in which to pose the Threshold Conjecture; indeed, see Theorem 1.4 .

1.5. Harmonic Yang-Mills connections on $\mathbb{R}^{4}$. As discussed earlier, steady states for the hyperbolic Yang-Mills equation are called harmonic Yang-Mills connections and play an important role in our work. They solve the equation

$$
\mathbf{D}^{j} F_{k j}=0 \quad \text { in } \mathbb{R}^{4},
$$

and can be seen as critical points for the (spatial) energy functional

$$
\mathcal{E}_{e}(A)=\int_{\mathbb{R}^{4}}\left\langle F^{i j}, F_{i j}\right\rangle d x .
$$

It is a classical result that any finite energy harmonic Yang-Mills connection on $\mathbb{R}^{4}$ is smooth in a suitable gauge $[53,54$. On the other hand, the question of existence of finite energy harmonic Yang-Mills connections is best phrased in terms of the topological classes described above:

Theorem 1.3. The following properties hold for harmonic Yang-Mills connections:

(1) Within each topological class there exist energy minimizers. These are called instantons, and they come in two varieties, self-dual $F=\star F$ and anti-self-dual $F=-\star F$, depending on the topological class.

(2) In particular, there exists a unique (up to symmetry) minimal energy nontrivial harmonic Yang-Mills connection $Q$, which is necessarily an instanton, whose energy $E_{\mathrm{GS}}$ satisfies

$$
\mathcal{E}\left(Q_{\mathrm{GS}}\right)=\left|\chi\left(Q_{\mathrm{GS}}\right)\right| .
$$

(3) All nontrivial harmonic Yang-Mills connections $Q$ with energy $\mathcal{E}(Q) \leq 2 E_{\mathrm{GS}}$ are instantons and satisfy

$$
\mathcal{E}(Q)=|\chi(Q)|
$$

Parts (1) and (2) are classical. We remark that part (3), which follows from a recent result of 8 , is nontrivial due to existence of nonminimizing harmonic YangMills connections 39. We also note that harmonic Yang-Mills connections which are not energy minimizers no longer have to be self-dual or anti-self-dual. We refer to [32, Sections 1.8 and 6] for further discussion.

As a consequence of the above properties, it easily follows that in the class of topologically trivial connections, the threshold for nontrivial harmonic YangMills connections is $2 E_{\mathrm{GS}}$ rather than $E_{\mathrm{GS}}$. Based on the above discussion, we will call subthreshold data/solution any topologically trivial hyperbolic Yang-Mills data/solution with energy below $2 E_{\mathrm{GS}}$.

1.6. The problem of gauge fixing, the Yang-Mills heat flow, and the caloric gauge. We now turn to the problem of gauge fixing, which is fundamental for the analysis of the hyperbolic Yang-Mills equation. As we will see, the classical gauges (Lorenz, temporal, Coulomb, etc.) by themselves turn out to be inadequate for the problem at hand. We will instead rely on the so-called caloric gauge, which 
is, remarkably, defined with the help of the Yang-Mills heat flow (see (1.16)), the parabolic counterpart of the Yang-Mills equation.

Due to the gauge invariance property, the solutions to the hyperbolic Yang-Mills equation are a priori only defined as equivalence classes. In order to properly formulate the notions of well-posedness and scattering which appear in the Threshold Conjecture, this invariance has to be taken into account. Gauge fixing refers to a procedure of selecting a unique representative in each gauge-equivalence class; it is often achieved by adding an additional set of constraint equations, to be satisfied by the connection 1-form $A$. In choosing a gauge for the hyperbolic Yang-Mills equation, one is naturally led to pursue conflicting goals:

(i) Causality: The system should have finite speed of propagation.

(ii) Structure: The nonlinearity should exhibit null structure type cancellation.

(iii) Large data: The gauge should be well-defined for large data.

Historically, there are (at least) three gauges that have played a role in the study of the hyperbolic Yang-Mills evolution:

1. The Lorenz gauge,

$$
\partial^{\alpha} A_{\alpha}=0
$$

In this gauge the Yang-Mills equation becomes a system of semilinear wave equations for $A_{\alpha}$, and in particular it has finite speed of propagation. This gauge is very convenient for local well-posedness for large but regular data. However, it is not so good in the low regularity setting as it does not capture well the null structure; see, e.g., 37.

2. The temporal gauge,

$$
A_{0}=0 .
$$

This again ensures that the above system is strictly hyperbolic, and in particular it has finite speed of propagation. In this gauge the equations can be understood as a semilinear wave equation for the curl of $A_{x}$, coupled with a transport equation for its divergence. This gauge is also very convenient for local well-posedness for large but regular data, and it fully describes all regular solutions to the hyperbolic Yang-Mills equation. Again, there are multiple technical difficulties if one tries to implement such a gauge in the low regularity setting or globally in time. In particular, we have no dispersion for the divergence of $A$. This gauge will play an auxiliary role in our analysis, and it is described in greater detail in section 4

3. The Coulomb gauge,

$$
\sum_{j=1}^{4} \partial_{j} A_{j}=0 .
$$

Here the causality is lost; however, the Coulomb gauge is an "elliptic" gauge which captures well the null structure of the problem, and thus it works well in low regularity settings. Indeed, the Coulomb gauge was used in 20] to prove the small data result for this problem. Unfortunately, it seems that the Coulomb gauge cannot be implemented globally for large data, even after restricting to those below the ground state energy. Nevertheless, for expository purposes we do provide a brief review of the Coulomb gauge in the beginning of section 2 ,

For the reasons described above, these three gauges seem inadequate for the purpose of proving the Threshold Theorem (to be described below). Instead, we introduce a new gauge: 
4. The caloric gauge. This is defined via the Yang-Mills heat flow

$$
\partial_{s} A_{j}=\mathbf{D}^{\ell} F_{\ell j}
$$

which is a gradient flow for the energy (1.15). We say that a connection 1-form $a$ is in the caloric gauge if the Yang-Mills heat flow $A(s)$ with $A(s=0)=a_{j}$ exists globally for all $s \in \mathbb{R}^{+}=[0, \infty)$ and converges to 0 as $s \rightarrow \infty$ in a suitable topology (see also Definition 2.6).

As we will describe in more detail in section 2, the caloric gauge has the key property that it is globally defined for all subthreshold data. In addition, this gauge agrees with the Coulomb gauge to the leading order, so there are many similarities between the analysis in the caloric and Coulomb gauges.

1.7. Main results of [30 33]. The first goal of our four papers 30 33] is to provide a sharp, affirmative answer to the Threshold Conjecture:

Theorem 1.4 (Threshold Theorem for energy critical Yang-Mills equation). Global well-posedness and scattering hold for the energy critical hyperbolic Yang-Mills evolution in $\mathbb{R}^{4+1}$ for all topologically trivial initial data with energy below $2 E_{\mathrm{GS}}$.

The statement of this theorem should be understood as follows:

- For each smooth subthreshold initial data $(a, e)$, there exists a global smooth solution, which is unique up to gauge transformations.

- For each subthreshold data in $\dot{H}^{1} \times L^{2}$, there exists a solution $\left(A, \partial_{t} A\right) \in$ $C\left(\mathbb{R} ; \dot{H}^{1} \times L^{2}\right)$ which is the unique limit of smooth solutions up to gauge transformations.

- Global well-posedness and scattering are formulated in the caloric gauge; see Theorem 5.3 for more details.

Since scattering solutions are necessarily topologically trivial, we are justified in considering only the topologically trivial data in Theorem 1.4. This restriction, in view of Theorem 1.3 is the reason why our threshold is $2 E_{\mathrm{GS}}$ rather than just $E_{\mathrm{GS}}$.

The second goal of our four papers 30 33. is to also consider solutions which do not satisfy the topological and energy constraints of the Threshold Theorem. Then on the one hand, we know there exist solutions which blow up or are global but do not scatter; see [15, 34. On the other hand, scattering can only hold for topologically trivial solutions. Because of this, our second result offers a dichotomy:

Theorem 1.5 (Dichotomy Theorem for energy critical Yang-Mills equation). The energy critical hyperbolic Yang-Mills evolution in $\mathbb{R}^{4+1}$ is locally well-posed in the energy space. Further, one of the following two properties must hold for the maximal solution:

(i) The solution is topologically trivial, is global, and scatters at infinity.

(ii) The solution bubbles off a soliton either

(a) at a finite blow-up time, or

(b) at infinity.

Several remarks are in order:

- Local well-posedness is established in the temporal gauge by exploiting its connection with the caloric gauge and its causality property; see sections 3.7 and 4.3 . 
- By scattering at positive (resp. negative) infinity, we mean that for a sufficiently large $T>0$, the solution restricted to the the time interval $[T, \infty)$ (resp. $(-\infty,-T])$ can be gauge transformed in to the caloric gauge, in which it scatters in the same sense as Theorem 1.4.

- The two alternatives in Theorem 1.5 hold separately for positive and negative time. In other words we do not eliminate the scenario where, say, scattering holds for positive time while finite time blowup occurs for negative time.

To fully describe this result, we need to clarify the meaning of bubbling off. We do this in the two scenarios of finite time blow-up solutions and of global solutions.

(a) The finite time blow-up scenario: Let $t_{0}>0$ be the blow-up time (maximal existence time) for a finite energy Yang-Mills connection A. By energy conservation, finite speed of propagation, and the small data result there must exist a point $x_{0} \in \mathbb{R}^{4}$ so that energy concentrates in the backward blow-up cone centered at $\left(t_{0}, x_{0}\right)$, namely $C=\left\{\left|x-x_{0}\right|<t_{0}-t\right\}$, in the sense that

$$
\lim _{t \nearrow t_{0}} \mathcal{E}_{S_{t}}(A)>0
$$

where $S_{t}=C \cap\left(\{t\} \times \mathbb{R}^{4}\right)$.

In this context, we say that $A$ bubbles off a soliton at $\left(t_{0}, x_{0}\right)$ if there exists a sequence of points $\left(t_{n}, x_{n}\right) \rightarrow\left(t_{0}, x_{0}\right)$ and scales $r_{n}$ with the properties

(1) time-like concentration,

$$
\limsup _{n \rightarrow \infty} \frac{x_{n}-x_{0}}{\left|t_{n}-t_{0}\right|}=v, \quad|v|<1
$$

(2) below self-similar scale,

$$
\limsup _{n \rightarrow \infty} \frac{r_{n}}{\left|t_{n}-t_{0}\right|}=0
$$

(3) convergence to soliton,

$$
\lim _{n \rightarrow \infty} r_{n} \mathcal{G}\left(O_{n}\right) A\left(t_{n}+r_{n} t, x_{n}+r_{n} x\right)=L_{v} Q(t, x) \quad \text { in } H_{\mathrm{loc}}^{1}\left([-1 / 2,1 / 2] \times \mathbb{R}^{4}\right),
$$

for some sequence of admissible gauge transformations $O_{n}$, a Lorentz transformation $L_{v}$, and finite energy harmonic Yang-Mills connection $Q$.

We remark that for a nontrivial finite energy harmonic Yang-Mills connection $Q$ we must have

$$
\mathcal{E}(Q) \leq \mathcal{E}\left(L_{v} Q\right)
$$

with equality if and only if $v=0$.

(b) Global solutions. Here we consider a finite energy Yang-Mills connection $A$ which is global forward in time. We say that $A$ bubbles off a soliton at infinity if there exists a sequence of points $C \ni\left(t_{n}, x_{n}\right) \rightarrow \infty$ and scales $r_{n}$ with the properties

(1) time-like concentration,

$$
\limsup _{n \rightarrow \infty} \frac{x_{n}}{t_{n}}=v, \quad|v|<1
$$

(2) below self-similar scale,

$$
\limsup _{n \rightarrow \infty} \frac{r_{n}}{t_{n}}=0
$$


(3) convergence to soliton,

$\lim _{n \rightarrow \infty} r_{n} \mathcal{G}\left(O_{n}\right) A\left(t_{n}+r_{n} t, x_{n}+r_{n} x\right)=L_{v} Q(t, x) \quad$ in $H_{\mathrm{loc}}^{1}\left([-1 / 2,1 / 2] \times \mathbb{R}^{4}\right)$,

for some sequence of admissible gauge transformations $O_{n}$, a Lorentz transformation $L_{v}$ and finite energy harmonic Yang-Mills connection $Q$.

1.8. A brief remark on prior works. We finally remark that these papers build upon a large body of work. This begins with early results on Yang-Mills above scaling 2, 3, 12, 14, 24, where the structure of the equations was first understood and exploited. Our general approach broadly follows the outline of similar results for wave maps, starting with the small data problem, the null frame function spaces, and the renormalization idea [44,51,52] and continuing with the induction on energy based energy dispersion approach in the proof of the Threshold and Dichotomy Theorem in [40,41] (see also [17] and [46, 50]). Similar results for the closely related massless Maxwell-Klein-Gordon equation at critical regularity were proved in the small data case in $[35](d \geq 6)$ and $[19](d \geq 4)$, respectively large data in [27 29] and independently in 16. Finally, the small data results for Yang-Mills were obtained only recently in [18] $(d \geq 6)$ and $[20](d \geq 4)$. For a more extensive overview of related literature, we refer the reader to [33. Some further comments on the literature are provided in each of the following sections as needed.

1.9. Outline of the article. The remainder of this article is devoted to an overview of the sequence of papers [30, 31, [32, and 33. These contain conceptually disjoint, self-contained logical steps which address different aspects of the problem, as follows:

I. The caloric gauge [30]: This first paper uses the Yang-Mills heat flow in order to introduce the caloric gauge, which is central in our analysis. Its main outcome is to provide a complete caloric gauge representation for the hyperbolic Yang-Mills equation (1.10). Along the way, we also establish the Threshold and the Dichotomy Theorems for the Yang-Mills heat flow. In particular, the former allows us to prove that all subthreshold data admit a caloric representation. These results are discussed in section 2 ,

II. Energy dispersed solutions [31]: Here we develop the analytic tools which are needed in order to understand the hyperbolic Yang-Mills flow in the caloric gauge. The main result is a strong quantitative a priori bound for energy dispersed solutions, which in particular implies local well-posedness as well as small data global well-posedness in the caloric gauge. The notion of energy dispersion as well as the main results are described in section 3 .

III. Large data and causality [32]: Since not all Yang-Mills solutions can be placed in the caloric gauge, in this article we show how to switch the qualitative part of the analysis (but not the analytic part) into the temporal gauge, in order to be able to deal with data with above threshold energy. The overview in section 4 also covers topological classes, initial data surgery, and gauge matters such as patching of local solutions.

IV. Blow-up analysis 33: In this final step we use Morawetz type bounds in order to perform a blow-up analysis which leads to the proof of the two theorems above. This is where the results in the previous two papers 31] 
and [33] are used, but not the the analysis leading to these results. This is described in section 5 .

\section{The CALORIC GAUGE}

This section describes the main results of 30, whose aim is to develop the caloric gauge as our main gauge of choice in the study of the hyperbolic Yang-Mills evolution.

Let us take as a starting point of our discussion the following small data result proved earlier in 20] (cf. Theorem 1.1):

Theorem 2.1. The hyperbolic Yang-Mills equation in $\mathbb{R}^{4+1}$ is globally well-posed in the Coulomb gauge for all initial data with small energy.

Unfortunately, while the Coulomb gauge works well in the small data problem, it does not appear to work for large data, even after restricting to only subthreshold data. This large data difficulty with the Coulomb gauge compels us to look for a different gauge choice, in which the Yang-Mills equation exhibits a similar null structure as the Coulomb gauge, yet which can be used in the large data problem.

Our solution to this problem is to introduce and use the (global) caloric gauge, which is constructed with the help of the Yang-Mills heat flow. A more localized form of this gauge was previously introduced by the first author in [25, 26, in order to study local well-posedness questions for the $(3+1)$-dimensional hyperbolic YangMills equation. This was in turn inspired by Tao's caloric gauge for wave maps [45, which is based on the harmonic map heat flow.

On the one hand, the caloric gauge resembles the Coulomb gauge in the sense that a generalized Coulomb condition holds (to be discussed in more detail in section 2.4). On the other hand, it can be used for a larger class of connections, which in particular includes all subthreshold connections (essentially by the Threshold Theorem for the Yang-Mills heat flow, see Theorem 2.4). Therefore, it furnishes a natural setting to state and prove the Threshold Theorem for the hyperbolic Yang-Mills equation; see Theorem 5.3.

2.1. The Coulomb gauge and the null structure. Before we describe the caloric gauge, we first review the null structure of the hyperbolic Yang-Mills equation in the Coulomb gauge, which plays an essential role in low regularity problems for the Yang-Mills equation.

Consider the expansion of the Yang-Mills equation (1.10) in terms of $A$, which takes the form

$$
\square A_{\beta}+2\left[A_{\alpha}, \partial^{\alpha} A_{\beta}\right]=\partial_{\beta} \partial^{\alpha} A_{\alpha}-\left[\partial^{\alpha} A_{\alpha}, A_{\beta}\right]+\left[A^{\alpha}, \partial_{\beta} A_{\alpha}\right]-\left[A^{\alpha},\left[A_{\alpha}, A_{\beta}\right]\right],
$$

where $\square_{A}:=\mathbf{D}^{\alpha} \mathbf{D}_{\alpha}$ is the covariant d'Alembertian (or the covariant wave operator). Separating the spatial part and the temporal part of the connection, one immediately sees that the spatial divergence of the solutions plays a prominent role. Precisely, one can rewrite the equations in the form

$$
\begin{aligned}
& \square_{A} A_{j}=\partial_{j} \partial^{k} A_{k}+\partial_{j} \partial^{0} A_{0}+\left[A^{\alpha}, \partial_{j} A_{\alpha}\right], \\
& \Delta_{A} A_{0}=\partial_{0} \partial^{j} A_{j}+\left[A^{j}, \partial_{0} A_{j}\right] .
\end{aligned}
$$


Thus, when imposing the Coulomb gauge condition

$$
\sum_{j=1}^{4} \partial_{j} A_{j}=0,
$$

the above equations turn into a hyperbolic system for the main variables

$$
\square_{A} A_{j}=\partial_{j} \partial^{0} A_{0}+\left[A^{\alpha}, \partial_{j} A_{\alpha}\right] .
$$

In order to eliminate the first term on the right and also to restrict the evolution to divergence-free fields $A_{j}$, we apply the Leray projection $\mathbf{P}$ and rewrite the equation in the form

$$
\square A_{j}=\mathbf{P}\left(\left[A^{\alpha}, \partial_{j} A_{\alpha}\right]-2\left[A^{\alpha}, \partial_{\alpha} A_{j}\right]-\left[\partial_{0} A_{0}, A_{j}\right]-\left[A^{\alpha},\left[A_{\alpha}, A_{j}\right]\right]\right),
$$

Here the $A_{0}$ component plays an auxiliary role, and is determined at each fixed time via the elliptic equation

$$
\Delta_{A} A_{0}=\left[A^{j}, \partial_{0} A_{j}\right] .
$$

This does not yet yield a self-contained system, as the time derivative of $A_{0}$ also appears in the first equation. A slightly more involved computation yields the equation

$$
\partial^{j} \mathbf{D}_{j} \mathbf{D}^{0} A_{0}=\partial^{j}\left(2\left[A_{0}, \partial^{0} A_{j}\right]+\left[\partial_{j} A_{\alpha}, A^{\alpha}\right]+\left[A_{\alpha},\left[A^{\alpha}, A_{j}\right]\right]\right),
$$

which also serves to determine $\mathbf{D}^{0} A_{0}$ in an elliptic fashion.

As one can easily see above, the Yang-Mills equations in the Coulomb gauge can be viewed as an evolution equation (2.4) for the spatial part $A_{x}$ of the connection, whereas $A_{0}$ and $\mathbf{D}^{0} A_{0}$ play the role of auxiliary, dependent variables. All terms in the equation which involve $A_{0}$ can be thought of as having more of an elliptic character and, to a large extent, have a perturbative nature. The quadratic terms

$$
\mathbf{P}\left(\left[A^{k}, \partial_{j} A_{k}\right]-2\left[A^{k}, \partial_{k} A_{j}\right]\right)
$$

can be thought of as the leading part of the nonlinearity. It is crucial that these terms satisfy the cancellation property known as the null condition.

As mentioned before, the Coulomb gauge works well for the small data problem (Theorem 2.1). Concerning large data, however, one sees here that in order to properly set up the Yang-Mills equation in the Coulomb gauge one would need to be able to invert the operator $\partial^{j} \mathbf{D}_{j}$. Exactly the same operator arises when one considers the linearization of the Coulomb gauge condition. This works well in the small data problem, but not so well for the large data problem.

2.2. Local and global theory for the Yang-Mills heat flow. Neglecting for the moment the time component of the connection $A$, at fixed time we consider the energy functional

$$
\mathcal{E}_{e}\left(A_{x}\right)=\frac{1}{2} \int_{\mathbb{R}^{4}}\left\langle F_{i j}, F^{i j}\right\rangle d x .
$$

The Yang-Mills heat flow is the gradient flow associated to this functional, which has the expression

$$
\partial_{s} A_{i}=\mathbf{D}^{\ell} F_{\ell i}, \quad A_{i}(s=0)=a_{i} .
$$

As written, this system is invariant with respect to purely spatial gauge transforms. To better frame the discussion, we observe that one can add a heat time component 
to the connection $A$ and rewrite the Yang-Mills heat flow in a fully covariant fashion as

$$
F_{s i}=\mathbf{D}^{\ell} F_{\ell i} .
$$

Then one can view the Yang-Mills heat flow equations in (2.7) as the effect of a gauge choice

$$
A_{s}=0
$$

(which we call the local caloric gauge) applied to the fully covariant Yang-Mills heat flow. This is akin to using the temporal gauge for the hyperbolic Yang-Mills equation.

We start with the basic result:

Theorem 2.2. The problem (2.7) is locally well-posed for data a $\in \dot{H}^{1}$.

The assumption $a \in \dot{H}^{1}$ restricts $a$ (and thus the solution) to the topologically trivial class. This is natural in view of our goal of constructing the caloric gauge and also for the eventual application to the Threshold Theorem (Theorem 1.4).

In the study of (2.7), a key role is played by the $L_{s, x}^{3}$-norm of the curvature $F_{i j}$. Precisely, the solution to (2.7) can be continued, and uniform covariant parabolic estimates for the solution can be proved for as long as $\|F\|_{L^{3}}$ remains finite. This motivates the following definition for the caloric size of a connection $a$ :

$$
\mathcal{Q}(a)=\left\{\begin{array}{cl}
\int_{\mathbb{R}^{+} \times \mathbb{R}^{4}}|F(s, x)|^{3} d s d x & \text { if the solution to (2.7) is global, } \\
\infty & \text { otherwise. }
\end{array}\right.
$$

We note that this is a scaling- and gauge-invariant quantity.

As described below, the caloric gauge is defined only for connections $a$ for which $\mathcal{Q}(a)$ is finite. This is an open subset of $\dot{H}^{1}$, as $\mathcal{Q}(a)$ has a locally Lipschitz dependence on $a$ whenever finite. Furthermore, for such $a$ we can describe the behavior of its Yang-Mills heat flow at infinity as follows:

Theorem $2.3([30])$. Let $a \in \dot{H}^{1}$ be a connection so that $\mathcal{Q}(a)<\infty$. Then the corresponding solution has the property that the limit

$$
\lim _{s \rightarrow \infty} A(s)=a_{\infty}
$$

exists in $\dot{H}^{1}$. Further, the limiting connection is flat, $f_{\infty}=0$.

The main technical difficulty with (2.7) is that it is only degenerate parabolic. Precisely, (2.7) can be formally viewed as a coupling of a strongly parabolic system for $F$ (which we think of as the curl of $A$ ) and a transport equation for the divergence of $A$.

We note that there is an alternate gauge choice which circumvents this issue, namely the de Turck gauge

$$
A_{0}=\partial^{j} A_{j}
$$

where the Yang-Mills heat flow becomes strongly parabolic and is easier to solve locally. In our formalism, the classical de Turck trick of compensating the degeneracy by a suitable $s$-dependent gauge transformation amounts to solving (2.8) in this gauge, hence the name.

Unfortunately, the transition from local to global is impossible in the de Turck gauge; in other words, in the de Turck gauge, Theorem 2.3 is false. One can see this by considering the evolution of flat connections. This is trivial under the local 
caloric gauge, but yields a $(4+1)$-dimensional harmonic heat flow for maps into $\mathbf{G}$ in the de Turck gauge, which is known to possibly blowup.

Our approach is instead based on a version of the de Turck trick for the linearization of (2.7) (namely, (2.12) below). In this scheme, an auxiliary flow called the dynamic Yang-Mills heat flow plays a major role. We will return to discussion of this idea in section 2.6 .

For now, we proceed to describe our next result proved in 30, which asserts that all connections with energy below threshold $2 E_{\mathrm{GS}}$ have finite caloric size, and thus Theorem 2.3 applies:

Theorem 2.4 (Threshold Theorem for the heat flow). There exists a nondecreasing function

$$
\mathcal{Q}:\left[0,2 E_{\mathrm{GS}}\right) \rightarrow[0, \infty)
$$

so that for every connection 1 -form $a \in \dot{H}^{1}$ with subthreshold energy $\mathcal{E}<2 E_{\mathrm{GS}}$, we have

$$
\mathcal{Q}(a) \leq \mathcal{Q}(\mathcal{E})
$$

This is proved using a concentration compactness type argument. The key ingredient is the energy monotonicity formula

$$
\mathcal{E}_{e}\left(A\left(s_{1}\right)\right)-\mathcal{E}_{e}\left(A\left(s_{2}\right)\right)=-\int_{s_{1}}^{s_{2}} \int\left\langle\mathbf{D}^{\ell} F_{\ell j}, \mathbf{D}^{k} F_{k}{ }^{j}\right\rangle d x d s .
$$

This formula yields good control of $A$ in the local caloric gauge, but not in the de Turck gauge. The same argument also gives the corresponding Dichotomy Theorem:

Theorem 2.5 (Dichotomy Theorem for the heat flow). For any $a \in \dot{H}^{1}$, one of the following two properties must hold for the maximally extended solution:

(i) The solution is topologically trivial and global and $\mathcal{Q}(a)<\infty$.

(ii) The solution bubbles off a harmonic Yang-Mills connection either

(a) at a finite blow-up time, or

(b) at infinity.

The bubbling argument here has roots in the classical work of Struwe 42 (see also Schlatter [36]) on compact manifolds. In comparison, the significance of the above theorems lies in the precise asymptotics of the Yang-Mills heat flow on the noncompact space $\mathbb{R}^{4}$, which allows us to construct the caloric gauge.

2.3. Caloric connections and the caloric manifold. Since the limiting connection $a_{\infty}$ given by Theorem 2.3 is flat, it must be gauge equivalent to the zero connection. Precisely, there exists a gauge transformation $O$ with the property that

$$
a_{\infty, j}=O^{-1} \partial_{j} O .
$$

Here $O=O(a) \in \dot{H}^{2}$ (interpreted in the sense that $O_{; j}:=\partial_{j} O O^{-1} \in \dot{H}^{1}$ ) is unique up to constant gauge transformations. Conjugating the full heat flow with respect to such an $O$ yields a gauge equivalent connection

$$
\tilde{A}_{j}=O A_{j} O^{-1}-O_{; j},
$$

which solves the Yang-Mills heat flow and satisfies $\tilde{a}_{\infty}=0$. This leads us to the following definition of caloric connections (see the end of section 1.6):

Definition 2.6. We will say that a connection $a \in \dot{H}^{1}$ is caloric if $a_{\infty}=0$. We denote the set of all such connections by $\mathcal{C}$. 
Theorem 2.4 can then be restated as an existence result for gauge equivalent caloric connections:

Theorem 2.7 ([30]). For every connection $a \in \dot{H}^{1}$ with $\mathcal{Q}(a)<\infty$, there exists a gauge equivalent caloric connection $\tilde{a} \in \dot{H}^{1}$, which is unique up to constant gauge transformations. In particular, this conclusion holds for all subthreshold connections.

The connection $\tilde{a}$ is defined as

$$
\tilde{a}_{j}=O a_{j} O^{-1}-O_{; j}, \quad O=O(a) .
$$

We note that the two connections have the same caloric size, $\mathcal{Q}(a)=\mathcal{Q}(\tilde{a})$.

To solve the Yang-Mills equation in the caloric gauge, we need to view the family $\mathcal{C}$ of the caloric gauge connections with energy below the ground state energy as an infinite-dimensional manifold. Here the $\dot{H}^{1}$ topology is no longer sufficient, so we introduce the slightly stronger topology

$$
\mathbf{H}=\left\{a \in \dot{H}^{1}: \partial^{j} a_{j} \in \ell^{1} L^{2}\right\}
$$

which reflects the fact (to be discussed later in more detail) that caloric connections satisfy a generalized, nonlinear form of the Coulomb gauge condition. Then we have

Theorem 2.8 (30]). For any caloric subthreshold connections a with energy $\mathcal{E}$ and caloric size $\mathcal{Q}$, we have the $\mathbf{H}$ bound

$$
\|a\|_{\mathbf{H}} \lesssim \mathcal{E}, \mathcal{Q} 1 .
$$

The set $\mathcal{C}$ of all $\dot{H}^{1}$ caloric connections is a $C^{1}$ infinite-dimensional submanifold of H.

We denote

$$
\tilde{a}=\operatorname{Cal}(a) .
$$

For arbitrary subthreshold $a \in \dot{H}^{1}$, this is only defined as an equivalence class, modulo constant conjugations. However, if in addition we know that $a \in \mathbf{H}$, then $O(a)$ is continuous, and we can fix its choice by imposing the additional condition

$$
\lim _{x \rightarrow \infty} O(x)=\mathrm{Id} .
$$

With this choice we have the following regularity property:

Theorem 2.9. The map $a \rightarrow O(a)$ is continuous (though not Lipschitz) from $\dot{H}^{1}$ to $\dot{H}^{2} 5$ It is also locally $C^{1}$ from $\mathbf{H}$ to $\dot{H}^{2} \cap C^{0} 6$

2.4. The tangent space and caloric data sets. Finite energy caloric YangMills waves will be continuous functions of time which take values into $\mathcal{C}$. They are however not smooth in time; instead, their time derivative will merely belong to $L^{2}$. Because of this, we need to take the closure of its tangent space $T \mathcal{C}$ (which a priori is a closed subspace of $\mathbf{H}$ ) in $L^{2}$. This is denoted by $T_{a}^{L^{2}} \mathcal{C}$. It is also convenient to have a direct way of characterizing this space; that is naturally done via the linearization of the caloric flow:

\footnotetext{
${ }^{5}$ Here $\dot{H}^{2}$ needs to be interpreted as a quotient space, modulo constant conjugations

${ }^{6}$ Here the action of the group of constant conjugations can be eliminated by using the condition (2.11).
} 
Definition 2.10. For a caloric gauge connection $a \in \mathcal{C}$, we say that $L^{2} \ni b \in T_{a}^{L^{2}} \mathcal{C}$ if the solution to the linearized local caloric gauge Yang-Mills heat flow equation

$$
\partial_{s} B_{k}=\left[B^{j}, F_{k j}\right]+\mathbf{D}^{j}\left(\mathbf{D}_{k} B_{j}-\mathbf{D}_{j} B_{k}\right), \quad B_{k}(0)=b_{k},
$$

satisfies

$$
\lim _{s \rightarrow \infty} B(s)=0 .
$$

Turning our attention now to the Yang-Mills heat flow, we will now consider solutions which at any fixed time $t$ are in the caloric gauge, $A_{x}(t) \in C$.

Definition 2.11. An initial data for the Yang-Mills equation in the caloric gauge is a pair $(a, b)$ where $a \in \mathcal{C}$ and $b_{k} \in T_{a}^{L^{2}} \mathcal{C}$.

The transition from one time to another requires understanding the linearization of the Yang-Mills heat flow. As in the Coulomb gauge, we will consider the spatial component of the connection as the dynamic variable and will view the temporal part of the connection as an auxiliary variable.

We begin our discussion by considering the initial data. To connect a general initial data $\left(a_{k}, e_{k}\right)$ with caloric initial data, we have the following result:

\section{Theorem 2.12.}

(1) For any initial data pair $(a, e) \in \dot{H}^{1} \times L^{2}$ with finite caloric size, there exists a caloric gauge data set $(\tilde{a}, b) \in T^{L^{2}} \mathcal{C}$ and $a_{0} \in \dot{H}^{1}$, unique up to constant gauge transformations and with continuous dependence in this quotient topology, so that $(\tilde{a}, \tilde{e})$ is gauge equivalent to $(a, e)$ and

$$
\tilde{e}_{k}=b_{k}-\left(\mathbf{D}_{\tilde{a}}\right)_{k} a_{0} .
$$

(2) For any caloric gauge initial data set $(\tilde{a}, b) \in T^{L^{2}} \mathcal{C}$, there exists a unique $a_{0} \in \dot{H}^{1}$, with Lipschitz dependence on $(a, b) \in \dot{H}^{1} \times L^{2}$, so that

$$
e_{k}=b_{k}-\left(\mathbf{D}_{a}\right)_{k} a_{0}
$$

satisfies the constraint equation (1.13).

In view of this result, we can fully describe caloric Yang-Mills waves as continuous functions

$$
I \ni t \rightarrow\left(A_{x}(t), \partial_{0} A_{x}(t)\right) \in T^{L^{2}} \mathcal{C} .
$$

An important role in the proof of this theorem is played by the following nonlinear div-curl type decomposition for the tangent space $T_{a}^{L^{2}} \mathcal{C}$ :

Theorem 2.13. Let $a \in \mathcal{C}$ with energy $\mathcal{E}$ and caloric size $\mathcal{Q}$. Then for each $e \in L^{2}$ there exists a unique decomposition

$$
e=b-\mathbf{D} a_{0}, \quad b \in T_{a}^{L^{2}} \mathcal{C}, \quad a_{0} \in \dot{H}^{1},
$$

with the corresponding bound

$$
\|b\|_{L^{2}}+\left\|a_{0}\right\|_{\dot{H}^{1}} \lesssim_{\mathcal{E}, \mathcal{Q}}\|e\|_{L^{2}} .
$$

Proving the latter theorem, in turn, requires understanding of the linearized equation (2.12); we will return to this issue in section 2.6. 
2.5. The dynamic Yang-Mills heat flow and the hyperbolic Yang-Mills equation. To proceed further, given a caloric Yang-Mills wave on $I$, we seek to interpret the (covariant) hyperbolic Yang-Mills equation

$$
\mathbf{D}^{\alpha} F_{\alpha \beta}=0,
$$

as gauge-dependent evolutions for $A_{\beta}$. Separating these equations into

$$
\mathbf{D}^{\alpha} \mathbf{D}_{\alpha} A_{k}=\mathbf{D}^{k} \mathbf{D}^{\alpha} A_{\alpha}-\left[A^{\alpha}, \mathbf{D}_{k} A_{\alpha}\right]
$$

respectively,

$$
\mathbf{D}^{k} \mathbf{D}_{k} A_{0}=\mathbf{D}_{0} \mathbf{D}^{k} A_{k}-\left[A^{k}, \mathbf{D}_{0} A_{k}\right]
$$

we seek to interpret the first equation as a hyperbolic evolution for $A_{x}$ and the second as an elliptic compatibility condition for $A_{0}$. This is achieved in several steps as follows:

(i) First, we show that the pair $\left(A_{x}, \partial_{0} A_{x}\right) \in T^{L^{2}} \mathcal{C}$ satisfies a generalized Coulomblike condition,

$$
\partial^{k} A_{k}=\mathbf{D A}(A), \quad \partial^{k} A_{k}=\mathbf{D B}(A, B),
$$

where DA and DB are nice maps on $T^{L^{2}} \mathcal{C}$, which contains an explicitly computed quadratic part as well as purely perturbative higher-order terms. Of course, this step does not have to anything to do with (2.15) and holds for any pair in $T^{L^{2}} \mathcal{C}$. The key computation for $\partial^{k} A_{k}$ is

$\partial^{k} A_{k}=-\int_{0}^{\infty} \partial^{k} \partial_{s} A_{k}(s) d s=-\int_{0}^{\infty} \mathbf{D}^{k} F_{s k}(s)+$ (quadratic and higher),

but by (2.7) the linear term vanishes. A similar computation holds for $\partial^{k} B_{k}$.

(ii) Next, we use the $\beta=0$ part of equation (2.15) to show that $A_{0}$ is uniquely determined by $A_{x}$ and $B_{x}=\partial_{0} A_{x}$, i.e.,

$$
A_{0}=\mathbf{A}_{0}\left(A_{x}, B_{x}\right),
$$

where $\mathbf{A}_{0}$ is a nice smooth map on $T^{L^{2}} \mathcal{C}$ which contains an explicitly computed quadratic part as well as purely perturbative higher-order terms.

(iii) Moreover, we use the $\beta \neq 0$ part of the equation (2.15) to show that $\mathbf{D}^{0} A_{0}$ is uniquely determined by $A_{x}$ and $B_{x}=\partial_{0} A_{x}$,

$$
\mathbf{D}^{0} A_{0}=\mathbf{D A}_{0}\left(A_{x}, B_{x}\right),
$$

where $\mathbf{D} \mathbf{A}_{0}$ is a nice smooth map on $T^{L^{2}} \mathcal{C}$ which again contains an explicitly computed quadratic part, as well as purely perturbative higher-order terms.

The above steps allow us, just as in the case of the Coulomb gauge, to view the spatial part of the connection $\left(A_{x}, \partial_{0} A_{x}\right) \in T^{L^{2}} \mathcal{C}$ as the dynamical variable and $A_{0}, \partial_{0} A_{0}$ as dependent variables. Precisely, we can recast the equations (2.16) in the form

$$
\square_{A} A_{k}=\mathbf{P}\left[A_{x}, \partial_{k} A_{x}\right]+2 \Delta^{-1} \partial_{k} \mathbf{Q}\left(\partial^{\alpha} A_{x}, \partial_{\alpha} A_{x}\right)+R\left(A, \partial_{t} A\right),
$$


where $\left[A_{x}, B_{x}\right]$ is a shorthand for $\left[A^{\ell}, B_{\ell}\right]$ and $\mathbf{Q}$ is a symmetric bilinear form with symbol 7

$$
\mathbf{Q}(\xi, \eta)=\frac{\xi^{2}-\eta^{2}}{2\left(\xi^{2}+\eta^{2}\right)}
$$

Here on the right we have two explicit quadratic terms depending only on $A_{x}$ and its time derivative, both of which have a favorable null structure and a remainder higher-order term $R$, which admits favorable $L^{1} L^{2}$ bounds and thus only plays a perturbative role. However, in the covariant d'Alembertian on the left, we still have the coefficients $A_{0}$ and $\mathbf{D}_{0} A_{0}$, which are determined as above in terms of $A_{x}$ and $\partial_{t} A_{x}$ :

$$
\begin{aligned}
A_{0} & =\mathbf{A}_{0}\left(A_{x}, B_{x}\right)=\mathbf{A}_{0}^{2}\left(A_{x}, B_{x}\right)+\mathbf{A}_{0}^{3}\left(A_{x}, B_{x}\right), \\
\mathbf{D}_{0} A_{0} & =\mathbf{D} \mathbf{A}_{0}\left(A_{x}, B_{x}\right)=\mathbf{D} A_{0}^{2}\left(B_{x}, B_{x}\right)+\mathbf{D A}_{0}^{3}\left(A_{x}, B_{x}\right) .
\end{aligned}
$$

Here the quadratic terms $\mathbf{A}_{0}^{2}\left(A_{x}, B_{x}\right), \mathbf{D A}_{0}^{2}\left(A_{x}, B_{x}\right)$ are explicit translation invariant bilinear forms,

$$
\begin{aligned}
\mathbf{A}_{0}^{2}\left(A_{x}, B_{x}\right) & =\Delta^{-1}\left[A_{x}, B_{x}\right]+2 \Delta^{-1} \mathbf{Q}\left(A_{x}, B_{x}\right), \\
\mathbf{D A}_{0}^{2}\left(B_{x}, B_{x}\right) & =-2 \Delta^{-1} \mathbf{Q}\left(B_{x}, B_{x}\right) .
\end{aligned}
$$

The remainders $\mathbf{A}_{0}^{3}\left(A_{x}, B_{x}\right), \mathbf{D A}_{0}^{3}\left(A_{x}, B_{x}\right)$, however, are not explicit but satisfy favorable bounds. Of these only the quadratic part of $A_{0}$ plays a nonperturbative role.

Finally, $A_{x}$ is also subject to a compatibility condition

$$
\partial^{k} A_{k}=\mathbf{D A}(A):=\mathbf{Q}(A, A)+\mathbf{D A}^{3}(A),
$$

where $\mathbf{D A}^{3}$ is perturbative.

To study the small data problem, it would be sufficient to work with the equation (2.19). However, for the large data problem we also need to flow the wave equation in the parabolic direction, which in turn requires us to specify the $s$-evolution equation for $A_{0}$. Our choice is to use the dynamic Yang-Mills heat flow

$$
F_{s \alpha}=\mathbf{D}^{\ell} F_{\ell \alpha},
$$

which is the (covariant) Yang-Mills heat flow (2.8) adjoined with $F_{s 0}=\mathbf{D}^{\ell} F_{\ell 0}$.

For nonzero heat-times $s, 2.15$ now becomes

$$
\mathbf{D}^{\alpha} F_{\alpha \beta}(s)=w_{\alpha},
$$

where in general $w_{\alpha}$, called the Yang-Mills tension field, is nontrivial as the two flows (wave and heat) do not commute. Thus additional steps are needed:

(iv) We compute parabolic evolutions for $w_{\alpha}$, showing that at time $t$ they depend only on the data $A_{x}(t), \partial_{t} A_{x}(t)$ and of course on $s$,

$$
w_{\alpha}=\mathbf{w}_{\alpha}\left(A_{x}(t), \partial_{t} A_{x}, s\right) .
$$

Moreover, we separate $\mathbf{w}_{\alpha}$ into an explicit quadratic part and a higher-order term

$$
\mathbf{w}_{\alpha}(s)=\mathbf{w}_{\alpha}^{2}(s)+\mathbf{w}_{\alpha}^{3}(s),
$$

${ }^{7}$ Given a scalar-valued symbol $m(\xi, \eta)$, our definition of the associated bilinear multiplier is

$$
\iint e^{i x \cdot(\xi+\eta)} m(\xi, \eta)\left[\hat{A}_{x}(\xi), \hat{B}_{x}(\eta)\right] \frac{d \xi}{(2 \pi)^{4}} \frac{d \eta}{(2 \pi)^{4}} .
$$


where the latter is purely perturbative.

(v) Finally, we recalculate $A_{0}$ and $\mathbf{D}^{0} A_{0}$ to include the dependence on $w(s)$ and write the analogue of the equation (2.19) for $A_{x}(s)$,

$$
\begin{aligned}
\square_{A(s)} A_{k}(s)= & \mathbf{P}\left[A^{j}(s), \partial_{k} A_{j}(s)\right]+2 \Delta^{-1} \partial_{k} \mathbf{Q}\left(\partial^{\alpha} A^{j}(s), \partial_{\alpha} A_{j}(s)\right) \\
& +R\left(A(s), \partial_{t} A(s)\right)+\mathbf{P} \mathbf{w}_{k}^{2}(s)+R_{s}\left(A, \partial_{t} A\right) .
\end{aligned}
$$

The extra terms on the right are matched by a like contribution to the quadratic part of $A_{0}$, i.e., (2.20) is replaced by

$$
A_{0}(s)=\mathbf{A}_{0}^{2}(A(s), B(s))+\mathbf{A}_{0}^{3}(A(s), B(s))+\Delta^{-1} \mathbf{w}_{0}^{2}(A, B)+\mathbf{A}_{0 ; s}^{3}(A, B) .
$$

The $s$-dependent terms in the above equations depend on the original connection $A$ and not just on $A(s)$. However, they have the redeeming feature that they are concentrated at a single dyadic frequency $s^{-\frac{1}{2}}$.

The analysis of equation (2.26) is now very similar to that of (2.19), with the minor proviso that the quadratic terms in $\mathbf{w}$ in (2.26) and (2.27) have a very mild nonperturbative role, and they exhibit a null form type cancellation.

2.6. Remarks on the dynamic Yang-Mills heat flow. In 30 the dynamic Yang-Mills heat flow (2.24) plays a major role in our proofs in several different ways:

(i) As a gauge covariant smoothing flow for spacetime connections. This is the most direct interpretation: (2.24) was used in this capacity to fix the evolution of $w_{\mu}(s)$ in the preceding subsection.

(ii) As a tool to perform the "infinitesimal de Turck trick" for the linearized YangMills heat flow in the local caloric gauge. As alluded to earlier, our understanding of (2.7) is based on its linearization (2.12), which in turn is analyzed through a version of de Turck trick. It is implemented as follows, using (2.24) as a useful auxiliary tool:

- Given a one-parameter family of Yang-Mills heat flows $A_{j}(t, x, s)$ with data $a_{j}(t, x)\left(t \in I, x \in \mathbb{R}^{4}, s \in J\right)$, we add a $t$-component $A_{0}(t, x, s)$ and view it as a connection 1 -form on $I \times \mathbb{R}^{4} \times J$. In the $s$-direction, we then impose the dynamic Yang-Mills heat flow (2.24).

- Afterward, the key idea is to work with

$$
F_{0 j}=\partial_{t} A_{j}-\mathbf{D}_{j} A_{0} .
$$

As opposed to $\partial_{t} A_{j}$, which solves (2.12), $F_{0 j}$ has the advantage of obeying a nondegenerate covariant parabolic equation:

$$
\mathbf{D}_{s} F_{0 j}-\Delta_{A} F_{0 j}-2 \operatorname{ad}\left(F_{j}^{\ell}\right) F_{0 \ell}=0 .
$$

Solving this equation would determine $F_{0 j}$ from any data $F_{0 j}(s=0)=e_{j}$. We choose $e_{j}=\partial_{t} a_{j}$, which amounts to prescribing $a_{0}=0$. Then $A_{0}$ may be determined by integrating $\partial_{s} A_{0}=F_{s 0}=\mathbf{D}^{\ell} F_{\ell 0}$, and then we come back to the solution $\partial_{t} A$ of (2.12).

(iii) As a tool to obtain a useful representation of the projection to the caloric manifold. This is a variant of (ii). Previously, we chose to initialize $a_{0}=0$. When $a(t=0)$ is a caloric connection, another natural choice is to set $A_{0}(s=\infty)=0$, which amounts to requiring that the nearby $a(t)$ 's are also caloric. Integrating 
$\partial_{s} A_{0}=\mathbf{D}^{\ell} F_{\ell 0}$ from $s=\infty$ to 0 , we obtain

$$
a_{0}=-\int_{0}^{\infty} \mathbf{D}^{\ell} F_{\ell 0}(s) d s .
$$

By (2.28), we have

$$
e_{j}=\partial_{t} a_{j}-\mathbf{D}_{j} a_{0}
$$

Since $a(t)$ 's are caloric, $\partial_{t} a_{j}$ clearly belongs to $T_{a} \mathcal{C}$, whereas $\mathbf{D} a_{0}$ is a pure covariant gradient. This procedure proves Theorem 2.13, while yielding a useful representation formula (2.29).

\section{ENERGy DisPersed CALORIC YANG-Mills WAVES}

Our second article 31] is concerned with the hyperbolic Yang-Mills equation in the caloric gauge, namely the equation (2.19) with the auxiliary variables $A_{0}$ and $D_{0} A_{0}$ as in (2.20) and the constraints (2.23).

3.1. Main results in the caloric gauge. The first result is a local well-posedness result which uses the notion of $\epsilon$-energy concentration scale, defined as

$$
r_{c}^{\epsilon}[a, e]=\sup \left\{r: \sup _{x} \int_{B_{r}(x)}|f|^{2}+|e|^{2} d x \leq \epsilon^{2}\right\} .
$$

Then we have

Theorem 3.1 ([31]). There exists a positive nonincreasing function $\epsilon_{*}(\mathcal{E}, \mathcal{Q})$ so that for any initial data set $(a, e)$ with energy $\mathcal{E}$ and initial caloric size $\mathcal{Q}$, that the Yang-Mills equation in the caloric gauge is locally well-posed in $\dot{H}^{1} \times L^{2}$ on the time interval $\left[-r_{c}^{\epsilon_{*}}, r_{c}^{\epsilon_{*}}\right]$.

We omit here the precise meaning of well-posedness and instead refer the reader to Theorem 5.3 in the last section. Precisely, the conclusions of Theorem 5.3 hold restricted to the interval $\left[-r_{c}^{\epsilon_{*}}, r_{c}^{\epsilon_{*}}\right]$.

The second main result in 31 uses the notion of energy dispersion, first introduced in [40] in the wave maps context. For a connection $A$ on a time interval $I$, we define its energy dispersion as

$$
\|F\|_{\mathrm{ED}[I]}=\sup _{k} 2^{-2 k}\left\|P_{k} F\right\|_{L^{\infty} L^{\infty}[I]} .
$$

Note that this norm is invariant under scaling. The second main result reads as follows:

Theorem 3.2. There exists a positive nonincreasing function $\epsilon(\mathcal{E})$ and a nondecreasing function $M(\mathcal{E})$ such that if $A$ is a caloric Yang-Mills wave on I with energy $\mathcal{E}$ and initial caloric size $\mathcal{Q} \lesssim_{\mathcal{E}} 1$ so that $\|F\|_{\mathrm{ED}} \leq \epsilon(\mathcal{E})$, then $8\|A\|_{S^{1}[I]} \leq M(\mathcal{E})$ and $A$ can be continued (as a well-posed solution in the sense of Theorem 3.1) past finite endpoints of $I$.

We also note that the initial assumption on $\mathcal{Q}$ only serves to prevent it from being very large. With this assumption, we actually show that $\mathcal{Q}(A) \ll 1$ in the entire interval $I$. By Theorem 2.4 this assumption can be entirely omitted for subthreshold energies.

These theorems, or rather their contrapositives, can be considered as continuation criteria for the hyperbolic Yang-Mills equation in the caloric gauge. By

\footnotetext{
${ }^{8}$ The control norm $S^{1}$ will be described shortly.
} 
providing an accurate description of how singularities may occur, they furnish a starting point for the bubble extraction argument in 33 as will be explained in section 5 .

One disadvantage of using the caloric gauge (or the Coulomb gauge) is that causality is lost. To remedy this, we prove that the well-posedness property can be transferred from the caloric gauge to the temporal gauge $A_{0}=0$. As a result, we obtain:

Theorem 3.3. The hyperbolic Yang-Mills equation in $\mathbb{R}^{4+1}$ is globally well-posed in the temporal gauge for all initial data with small energy.

Unlike the caloric gauge results, however, a downside of Theorem 3.3 is that it does not provide the $S^{1}$ regularity of solutions or any other dispersive bounds.

In the remainder of this section, we will give an overview of ideas in the proofs of Theorems 3.1, 3.2, and 3.3 ,

3.2. Function spaces. To state the results more precisely and also to discuss their proof, it is necessary to outline the function spaces framework used in 31, whose main components are the same as in 19,20 . The core solution space, which we denote by $S^{1}[I]$, is a Banach space of functions on $I \times \mathbb{R}^{4}$ with the property that elements of $S^{1}[I]$ inherit estimates satisfied by free waves in the energy class (i.e., $\square u=0$ with $\left.\left(u, \partial_{t} u\right)(0) \in \dot{H}^{1} \times L^{2}\right)$, such as energy estimates, Strichartz estimates, (null form) bilinear estimates, etc. The corresponding nonlinearity space, denoted by $N[I]$, is defined, on the one hand, small enough to satisfy the inhomogeneous estimate

$$
\|u\|_{S^{1}[I]} \lesssim\left\|\left(u, \partial_{t} u\right)(0)\right\|_{\dot{H}^{1} \times L^{2}}+\|\square u\|_{N[I]},
$$

and on the other hand, large enough to contain (at least, most of) the nonlinearities of the wave equation (2.19).

Construction of these spaces builds up on many prior works. The space $N[I]$ is simply the sum of the dual energy space (i.e., $\left.L^{1} L^{2}[I]\right)$ and a dual $X^{s, b}$ space. Building blocks of the space $S^{1}[I]$ include the energy space (i.e., $\|\nabla u\|_{L^{\infty} L^{2}[I]}$ ), the Strichartz spaces (i.e., $\left\||D|^{-\alpha} \nabla u\right\|_{L^{p} L^{q}[I]}$ with admissible $\alpha, p, q$ ), an $X^{s, b}$ space [1,11, the refined Strichartz spaces with radial frequency localization [14, and the null frame space [44,51]. Moreover, we also add a new component $S^{\text {sq }}$ (to be described in section 3.7), which is used in the proof of Theorem 3.3. For the precise definition, we refer to [31, Section 4].

The $S^{1}[I]$-norm serves the role of a controlling norm for the caloric Yang-Mills waves. More precisely, we show in [31] that finiteness of this norm implies finer properties of the solution itself and those nearby, such as frequency envelope control, persistence of regularity and scattering for $A_{x}$, as well as weak Lipschitz dependence and local-in-time continuous dependence for the nearby solutions. For details, see the structure theorems in [31, Section 4].

3.3. Truncated energy dispersion and the central result. It turns out that Theorems 3.1 and 3.2 can be proved essentially at the same time. The idea is to use smallness of the truncated energy dispersion at frequencies higher than $2^{m}$,

$$
\|F\|_{\mathrm{ED}>m[I]}=\sup _{k>m} 2^{-2 k}\left\|P_{k} F\right\|_{L^{\infty} L^{\infty}[I]}
$$

matched with shortness of the time interval on the scale $2^{-m}$. The central result of 31] reads as follows. 
Theorem 3.4. There exist a nondecreasing positive function $M(\mathcal{E}, \mathcal{Q})$ and nonincreasing positive functions $\epsilon(\mathcal{E}, \mathcal{Q})$ and $T(\mathcal{E}, \mathcal{Q})$, so that the following holds. For all regular subthreshold caloric Yang-Mills waves $A$ in a time interval I with energy $\mathcal{E}$ and initial caloric size $\mathcal{Q}$, if we have

$$
\|F\|_{\mathrm{ED}_{\geq m}[I]} \leq \epsilon(\mathcal{E}, \mathcal{Q}), \quad|I| \leq 2^{-m} T(\mathcal{E}, \mathcal{Q}),
$$

then we must also have

$$
\|A\|_{S^{1}[I]} \leq M(\mathcal{E}, \mathcal{Q}) .
$$

On the one hand, this theorem implies an $S^{1}[I]$-control norm bound on a time interval of size $\leq 2^{-m}$ for data with sufficiently small energy at frequencies $>2^{m}$ (i.e., $\left\|P_{>m}\left(A_{x}, \partial_{t} A_{x}\right)(0)\right\|_{\dot{H}^{1} \times L^{2}}$ is small), which is the case for data with energy concentration scale $\gtrsim 2^{-m}$. On the other hand, it also implies an $S^{1}[I]$-bound, independent of $I$, if the solution has small untruncated energy dispersion $\|F\|_{\operatorname{ED}[I]}$. As discussed above, these $S^{1}[I]$-norm bounds prove Theorems 3.1 and 3.2 , respectively.

\subsection{Review of the small energy case: Perturbative nonlinearities and} parametrix construction. We begin with a brief discussion of the small energy case, where the goal is to prove $\left\|A_{x}\right\|_{S^{1}[\mathbb{R}]}^{2} \leq C \mathcal{E}$ for sufficiently small $\mathcal{E}$. This was carried out in 20, which can be viewed as one of the predecessors to this work, in the closely related context of the Coulomb gauge 9

The first step was to try to view the wave equation for $A_{x}$ as a perturbation of the constant coefficient wave equation $\square A_{x}=0$. While this is not possible, we can view most of the nonlinearity as perturbative and estimate them in the space $N$. In this process, the primary (bilinear) null structure of the Yang-Mills equation, uncovered in [13, plays an essential role. This leaves us with a single nonperturbative term, which arises in a paradifferential fashion,

$$
\left(\square+\operatorname{Diff}_{\mathbf{P} A}^{0}\right) A_{x}:=\left(\square+2 \sum_{k} \operatorname{ad}\left(P_{<k} \mathbf{P}^{\alpha} A\right) \partial_{\alpha} P_{k}\right) A_{x}=G,
$$

where $\mathbf{P}_{x} A$ is the Leray projection of $A_{x}, \mathbf{P}_{0} A=A_{0}$ and $G$ represents a nonlinear but perturbative contribution (which is small thanks to smallness of energy).

Then the key step in 20] was to construct a parametrix for the paradifferential operator $\square+\operatorname{Diff}_{\mathbf{P} A}^{0}$ and to prove that this parametrix satisfies a $\operatorname{good} N \rightarrow S^{1}$ bound akin to (3.1). The rough idea is to try to find a gauge transform $O$ which renormalizes $\square+\operatorname{Diff}_{\mathbf{P} A}^{0}$ to $\square$ modulo a better behaved error, i.e., schematically

$$
\left(\square+\operatorname{Diff}_{\mathbf{P} A}^{0}\right) \operatorname{Ad}(O)-\operatorname{Ad}(O) \square=\text { (error), }
$$

and to produce a parametrix by conjugating the constant coefficient solution operator by $\operatorname{Ad}(O)^{-1}$.

This idea was indeed viable in the case of wave maps [40,44, but not for YangMills or Maxwell-Klein-Gordon (which may be regarded as a simpler model for Yang-Mills). The difference stems from the structure of the curvature $F[\mathbf{P} A]$, which is a geometric obstruction for gauge transformation of $A$ to 0 . Whereas the curvature depends at least quadratically on the solution in the case of wave maps, it

\footnotetext{
${ }^{9}$ While the analysis in 20] is carried out in the Coulomb gauge $\partial^{\ell} A_{\ell}=0$, it is not very different in the caloric gauge, as this also satisfies some form of generalized Coulomb condition $\partial^{\ell} A_{\ell}=\mathbf{D A}(A)$.
} 
is linear (to the leading order) in the solution $A$ for Yang-Mills or Maxwell-KleinGordon.

The way out of this difficulty was to consider instead an $\operatorname{Ad}(\mathbf{G})$-valued pseudodifferential renormalization operator $\operatorname{Op}(\operatorname{Ad}(O))$. Heuristically, this generalization allows for separate renormalization of each plane wave solution, which is possible since it only oscillates in a single direction 10 Using smallness of energy, it was shown that the parametrix obeys the desired $N \rightarrow S^{1}$ bound and also that the error in (3.6) is perturbative. We remark that in the error estimate, not only the primary but also the secondary (trilinear) null structure, analogous to that in Maxwell-Klein-Gordon discovered in 23, are crucial.

3.5. The parametrix construction in the large energy case. The difference in the large energy case is that we can no longer use smallness of energy to control neither the perturbative part nor the parametrix for the paradifferential problem. Thus, in order to be able to close our estimates, we need to have new proxies for smallness.

We start with the paradifferential problem. In a departure from the small energy case, but similar to [29,40], we introduce the large frequency gap $\kappa \gg 1$ and consider the paradifferential operator

$$
\square+\operatorname{Diff}_{\mathbf{P} A}^{\kappa}=\square+2 \sum_{k} \operatorname{ad}\left(P_{<k-\kappa} \mathbf{P}^{\alpha} A\right) \partial_{\alpha} P_{k},
$$

where $A_{x}$ is a caloric Yang-Mills wave with finite $S^{1}[I]$-norm. The goal is to establish an $N \rightarrow S^{1}$ bound of the form

$$
\|u\|_{S^{1}[I]} \lesssim\left\|A_{x}\right\|_{S^{1}[I]}\left\|\left(u, \partial_{t} u\right)(0)\right\|_{\dot{H}^{1} \times L^{2}}+\left\|\left(\square+\operatorname{Difff}_{\mathbf{P} A}^{\kappa}\right) u\right\|_{N[I]} .
$$

The proof proceeds via a parametrix construction, in a manner similar to 20 . However, the necessary smallness for proving the $N \rightarrow S^{1}$ bound for the parametrix now comes from taking the frequency gap $\kappa$ sufficiently large compared to $\left\|A_{x}\right\|_{S^{1}[I]}$. Moreover, in order to control the error, we rely on the divisibility 11 of an appropriate weaker norm $\left\|A_{x}\right\|_{D S^{1}[I]}$ than $\left\|A_{x}\right\|_{S^{1}[I]}$.

Treating the perturbative nonlinearity: Small energy dispersion and short time interval. For perturbative nonlinearity, smallness may be obtained via truncated energy dispersion and the length of $I$. Roughly speaking, any unbalanced or close-angle frequency interaction is small (exponentially in the frequency ratio) for such nonlinearities, while balanced and far-angle interactions are controlled by $\|F\|_{\mathrm{ED}_{>m}[I]}$ at frequencies $\gtrsim 2^{m}$ and by $2^{m}|I|$ at frequencies $\lesssim 2^{m}$. In sum, we have

$$
\|F\|_{E D>m[I]} \leq \varepsilon, \quad 2^{m}|I| \leq \varepsilon \Longrightarrow\left\|\left(\square+\operatorname{Diff}_{\mathbf{P} A}^{\kappa}\right) A_{x}\right\|_{N[I]} \lesssim\left\|A_{x}\right\|_{S^{1}[I]} 2^{C \kappa} \varepsilon^{\delta} .
$$

Unfortunately, this bound is insufficient for proving Theorem 3.4. The reason is that the $N \rightarrow S^{1}$ bound (3.7) for the paradifferential operator already depends on the $S^{1}[I]$-norm of $A_{x}$, which is what we wish to bound!

\footnotetext{
${ }^{10}$ This procedure bypasses the geometric obstruction mentioned above, since curvature, being a 2-form, always vanishes when restricted to a one-dimensional subspace.

${ }^{11}$ That is, $I$ can be split into a controlled number of subintervals, on each of which the restricted norm is arbitrarily small.
} 
3.6. Induction on energy. In order to break the circular argument, we perform an induction on energy, following the scheme developed in [40. Roughly speaking, the main idea is to view $A$ as a perturbation of another solution $\tilde{A}$, which has a lower (linear) energy and hence obeys an $S^{1}$-norm bound by an induction hypothesis. To make this idea work, we need to carefully construct $\tilde{A}$ so that we may control the difference $A-\tilde{A}$.

A preliminary step here is to show that $\mathcal{Q}$ is essentially conserved for solutions with small energy dispersion. Once this is done, $\mathcal{Q}$ becomes a fixed parameter and is omitted from the subsequent discussion.

The induction argument is set up as follows, in terms of the linear energy $E$ rather than the nonlinear one $\mathcal{E}$. The initial step is provided by the small energy case, which proves (3.4) up to sufficiently small $E>0$, with $M(E)=C \sqrt{E}$ and any choices of $\epsilon(E), T(E)$. As in the induction hypothesis, we assume that there exist functions $\epsilon(\cdot), T(\cdot)$, and $M(\cdot)$ such that (3.4) holds up to some $E$. Then the goal is to extend these functions so that (3.4) holds up to $E+c_{0}$ for some $c_{0}=c_{0}(E)>0$. An essential point for continuing this induction argument (in order to cover all subthreshold solutions) is to ensure that the increment $c_{0}(E)$ is independent of the functions $\epsilon(\cdot), T(\cdot)$, and $M(\cdot)$ given by the induction hypothesis 12

We define $\tilde{A}$ by first flowing the data $\tilde{A}_{x}(0)$ and $\partial_{t} \tilde{A}_{x}(0)$ by the Yang-Mills heat flow and the linearized Yang-Mills heat flow, respectively, for some heat-time $s_{*}$, then solving the Yang-Mills equation in caloric gauge in time. Taking $\epsilon, T$, and $c_{0}$ sufficiently small, and choosing $s_{*}$ appropriately, we aim for the following two goals:

(i) $\tilde{A}$ exists on $I$ and $\|\tilde{A}\|_{S^{1}[I]} \leq M(E)$;

(ii) $\|A-\tilde{A}\|_{S^{1}[I]} \lesssim_{M(E)} 1$.

The cutoff heat-time $s_{*}$ can be chosen so that either

(a) $s_{*} \ll 2^{-m}$ and $\|\nabla \tilde{A}(0)\|_{L^{2}}=E$, or

(b) $s_{*} \simeq 2^{-m}$ and $\|\nabla \tilde{A}(0)\|_{L^{2}} \geq E$.

In both cases, provided that $\epsilon, T$ are sufficiently small, it can be shown that $\tilde{A}_{x}$ is close to the Yang-Mills heat flow $A_{x}\left(s_{*}\right)$ of $A_{x}$. In case (a), taking $\epsilon$ smaller if necessary, we may ensure that $\|\tilde{F}\|_{\mathrm{ED}_{\geq m}} \leq \epsilon(E)$ and goal (i) follows from the induction hypothesis. In case (b), $\tilde{A}(0)$ is sufficiently smooth so that the desired conclusion can be proved simply by higher-order local well-posedness.

To accomplish goal (ii), we need several ideas. First, we observe that the linear energies $\left\|\nabla A_{x}(t)\right\|_{L^{2}},\left\|\nabla \tilde{A}_{x}(t)\right\|_{L^{2}}$ of the solutions $A, \tilde{A}$ are conserved in $t$, up to an error that can be made arbitrarily small by taking $\epsilon, T$ small enough. Moreover, since $\tilde{A}$ is close to $A\left(s_{*}\right)$, which in turn is (at least heuristically) a low frequency truncation of $A$, the frequency supports of $A-\tilde{A}$ and $\tilde{A}$ are essentially separated. Therefore, approximate conservation of linear energies for $A$ and $\tilde{A}$ implies

$$
\sup _{t \in I}\left\|\nabla\left(A_{x}-\tilde{A}_{x}\right)(t)\right\|_{L^{2}} \lesssim_{E}\left\|\nabla A_{x}(0)\right\|_{L^{2}}-\left\|\nabla \tilde{A}_{x}(0)\right\|_{L^{2}} \leq c_{0} .
$$

To upgrade this to an $S^{1}[I]$-norm bound, we establish a weak divisibility property of the $S^{1}$-norm of $\tilde{A}$, i.e., that we can split $I=\bigcup_{k=1}^{K} I_{k}$ so that

$$
\left\|\tilde{A}_{x}\right\|_{S^{1}\left[I_{k}\right]} \lesssim_{E} 1, \quad K \lesssim_{M(E)} 1 .
$$

\footnotetext{
${ }^{12}$ Meanwhile, $\epsilon=\epsilon\left(E+c_{0}\right), T=T\left(E+c_{0}\right)$, and $M=M\left(E+c_{0}\right)$ may (and indeed do) depend
} on $\epsilon(E), T(E)$, and $M(E)$. We are allowed to choose these parameters in the order $c_{0} \rightarrow M \rightarrow T, \epsilon$. 
Now viewing $A=\tilde{A}+(A-\tilde{A})$ as a perturbation of $\tilde{A}$ on each $I_{k}$, where the data for $A-\tilde{A}$ are reinitialized on each interval using (3.8), we may bound the $S^{1}$-norm of $A-\tilde{A}$ on each $I_{k}$ provided that $c_{0}$ is small enough compared to the implicit constants in (3.8) and (3.9). Importantly, these are independent of $M(E)$ ! Thus goal (ii) follows by summing up these bounds in $k=1, \ldots, K$.

3.7. Passing to the temporal gauge. Finally, we describe the ideas behind the proof Theorem 3.3. We wish to estimate the gauge tranformation $O$ from the caloric gauge into the temporal gauge, which solves the nonlinear transport equation

$$
O^{-1} \partial_{t} O=A_{0} \text {. }
$$

For $O$ to preserve $\dot{H}^{1}$ regularity of $A_{x}$, we need

$$
\Delta A_{0} \in \ell^{1} L_{x}^{2} L_{t}^{1} .
$$

The proof of (3.10) relies on two observations.

(i) We note that the following square function norm can be added to the $S^{1}$-norm, i.e.,

$$
\left\|\nabla A_{x}\right\|_{S^{\mathrm{sq}}} \lesssim\left\|A_{x}\right\|_{S^{1}}
$$

where

$$
\|u\|_{S^{\mathrm{sq}}}=\left\||D|^{-\frac{3}{10}} u\right\|_{\ell^{2} L_{x}^{\frac{10}{3}} L_{t}^{2}} .
$$

The relevance of $p=\frac{3}{10}$ is that it is the dual Stein-Tomas exponent for Fourier restriction to $\mathbb{S}^{3} \subseteq \mathbb{R}^{4}$. Indeed, the (adjoint) Stein-Tomas restriction theorem and Plancherel in time leads to

$$
\left\|e^{ \pm i t|D|} u\right\|_{S^{s q}} \lesssim\|u\|_{L^{2}}
$$

which implies $\nabla u \in S^{\mathrm{sq}}$ for $\dot{H}^{1}$ free waves. We extend this estimate to our parametrix, which allows us to add $S^{\text {sq }}$ into our $S^{1}$-norm.

(ii) In an order 0 bilinear expression of the form $\mathbf{O}\left(A_{x}, \partial_{t} A_{x}\right)$, the worst case is when $\partial_{t} A_{x}$ has the higher frequency. Indeed, the ordinary product $\left[A_{x}, \partial_{t} A_{x}\right]$ fails to belong to $\ell^{1} L_{x}^{2} L_{t}^{1}$ because of this interaction. However, from (2.21), we see that the symbol of $\Delta \mathbf{A}_{0}^{2}$ is

$$
\Delta \mathbf{A}_{0}^{2}(\xi, \eta)=\frac{2|\xi|^{2}}{|\xi|^{2}+|\eta|^{2}}
$$

which exhibits a favorable gain in the problematic low $\times$ high interaction!

\section{Large Data, CAUSAlity, AND the temporal GaUge}

Unlike the first two papers, the third one 32] is concerned with large data solutions which are not necessarily topologically trivial and, thus, cannot be directly studied using the global caloric gauge. The goal of [32] is twofold:

- to describe finite energy initial data sets topologically and analytically;

- to use the temporal gauge in order to provide a good local theory for finite energy solutions.

For simplicity we will work in two settings:

(a) for initial data in $\mathbb{R}^{4}$ and solutions in $\mathbb{R}^{4+1}$, or time sections thereof;

(b) for initial data in a ball $B_{R}$ and solutions in the corresponding uniqueness cone $\mathcal{D}\left(B_{R}\right)=\{|x|+|t|<R\}$ or time sections thereof. 
In terms of the initial data, in addition to the energy, a key role is played by the energy concentration scale13

$$
r_{c}^{\epsilon}=\sup \left\{r>0: \mathcal{E}_{B_{r}(x) \cap X}(a, e) \leq \epsilon \text { for all } x \in X\right\},
$$

where $X=B_{R}$ or $\mathbb{R}^{4}$, as well as the outer concentration radius

$$
R_{c}^{\epsilon}=\inf \left\{r>0: \mathcal{E}_{B(x, r)}(a, e) \leq \epsilon \text { for some } x \in \mathbb{R}^{4}\right\} .
$$

4.1. Initial data surgery. Here we discuss a technical tool introduced in 32, which may be of independent interest. At various points in the analysis, we need to perform a physical space localization of the Yang-Mills solution. By finite speed of propagation, this task amounts to smoothly cutting off an initial data set $(a, e)$, which turns out to be nontrivial due to the presence of the constraint equation (1.13). To address this issue, we prove the following result:

Theorem 4.1. Let $B=B_{R_{0}}(0)$ be a ball centered at 0 , and let a be an $\dot{H}^{1}$ connection on $\mathbb{R}^{4} \backslash B$. Then there exists a solution operator $h \mapsto e=T_{a} h$ to the equation

$$
\mathbf{D}^{\ell} e_{\ell}=h \quad \text { in } \mathbb{R}^{4} \backslash B
$$

with the following properties:

(1) Boundedness: The operator $T_{a}$ is bounded from $\dot{H}^{-1}$ to $L^{2}$, with a norm depending only on $\|a\|_{L^{4}}$.

(2) Higher regularity: If $a$ and $h$ are smooth, then $T_{a} h$ is also smooth.

(3) Exterior support: For any $R \geq R_{0}$, if $h=0$ in $B_{R}(0)$, then $T_{a} h=0$ in $B_{R}(0)$.

In the case $a=0$, (4.1) becomes the usual divergence equation, and a desired solution operator $T_{0}$ may be constructed explicitly. Exploiting the exterior support property of $T_{0}, T_{a}$ is constructed in an essentially inductive manner, starting from an annulus around $B$ (where $a$ can be treated perturbatively) and proceeding outward.

As a quick corollary of Theorem 4.1, we obtain the following initial data excision result.

Proposition 4.2. Let $(a, e)$ be a small energy data set in $B_{4} \backslash B_{1}$. Then

(1) We can find a small energy exterior data set $(\tilde{a}, \tilde{e})$ in $\mathbb{R}^{4} \backslash B_{1}$, which agrees with $(a, e)$ in $B_{2} \backslash B_{1}$. Furthermore, if $(a, e)$ is smooth then $(\tilde{a}, \tilde{e})$ is also smooth.

(2) We can find a small energy exterior data set $(\tilde{a}, \tilde{e})$ in $\mathbb{R}^{4} \backslash B_{1}$, which is gauge equivalent to $(a, e)$ in $B_{4} \backslash B_{2}$. Furthermore, if $(a, e)$ is smooth then $(\tilde{a}, \tilde{e})$ is also smooth.

The idea of the proof is to first naively extend $(a, e)$ to $\mathbb{R}^{4} \backslash B_{1}$. This generates an error in the constraint equation, which can be removed by applying Theorem 4.1,

Remark 4.3. Theorem 4.1 can clearly be generalized to other regularities and dimensions. In particular, the operator $T_{a}: \dot{H}^{-1}\left(\mathbb{R}^{3} \backslash B\right) \rightarrow L^{2}\left(\mathbb{R}^{3} \backslash B\right)$ can be used to prove an excision result for finite energy data on $\mathbb{R}^{3}$. We note that this furnishes an alternative approach to constructing local Coulomb gauges [13] that avoids the need to prescribe boundary values.

\footnotetext{
${ }^{13}$ For a singlet $a$, we define $r_{c}^{\epsilon}$ and $R_{c}^{\epsilon}$ by taking $e=0$.
} 
4.2. Good global gauges. In view of the gauge independence property, having control of the energy of a connection a says little about the $\dot{H}^{1} \cap L^{4}$ size of $a$. This issue can sometimes be addressed by choosing a good gauge, such as the local Coulomb gauge in Uhlenbeck's lemma for small energies or the caloric gauge for subthreshold energies; see Theorems 2.4, 2.8. However, what if our connection has larger energy?

We begin our discussion with initial data sets in a ball. In addition to the energy $\mathcal{E}$, we also use a second parameter, namely the energy concentration scale $r_{c}=r_{c}^{\epsilon}$, with a small universal constant $\epsilon$. Then we have

Proposition 4.4. Given a connection a in $B_{R}$ with energy $\mathcal{E}$ and energy concentration scale $r_{C}$, there exists a gauge equivalent connection $\tilde{a}$ in $B_{R}$ which satisfies the bound

$$
\|\tilde{a}\|_{\dot{H}^{1} \cap L^{4}} \lesssim_{E, \frac{r_{c}}{R}} 1 .
$$

For initial data in $\mathbb{R}^{4}$ we also can find a good global gauge:

Theorem 4.5 (Good global gauge). Let a $\in H_{\text {loc }}^{1}\left(\mathbb{R}^{4}\right)$ be a finite energy connection. Then there exists a gauge equivalent representation $\tilde{a}$ of a such that

$$
\tilde{a}=-\chi O_{(\infty) ; x}+b,
$$

where $O_{(\infty)}(x)$ is a smooth 0-homogeneous map taking values in $\mathbf{G}, b \in \dot{H}^{1}$, and $\chi$ is a smooth cut-off function which vanishes near the origin and equals 1 near infinity.

Finally, we remark on the relationship between Theorem 4.5 and topological classes of finite energy connections. Precisely, the topological class of a connection $a$ can be parametrized by the homotopy class $[O]$ of the map $O$ in the above theorem, viewed as a map

$$
O: \mathbb{S}^{3} \rightarrow \mathbf{G}
$$

4.3. The temporal gauge and causality. While we are not able to carry out the full analysis for the Yang-Mills equation in the temporal gauge, we are nevertheless making good use of it in our papers in an auxiliary role. This is due to the following three properties:

(i) local well-posedness for regular data;

(ii) causality, i.e., finite speed of propagation;

(iii) agreement with caloric gauge at the linear level.

In our sequence of papers we are taking advantage of these three properties at different places in the analysis. Property (i), for instance, is used in order to prove a local well-posedness for regular data in the caloric gauge, simply by gauge transforming the temporal solutions. Property (iii), essentially described in section 3.7. allows us to reverse the process and to show that small energy global well-posedness in the caloric gauge implies small energy global well-posedness in the temporal gauge. Finally, as a consequence of property (ii), the small energy global well-posedness in the temporal gauge implies large energy local well-posedness in the temporal gauge. Even better, it shows that the local solutions can be continued in the temporal gauge for as long as no energy concentration occurs in a light cone. 
4.3.1. Finite energy solutions. A consequence of [20] and of the first two papers in the series [30, [31] is that the small data problem for the $(4+1)$-dimensional hyperbolic Yang-Mills equation is well-posed in several gauges: Coulomb, caloric, and temporal. In 32 we exploit the temporal gauge small data result, combined with causality, to obtain results for the large data problem. The local-in-time result is as follows:

Theorem 4.6 ([32]).

(1) For each finite energy data $(a, e)$ in $\mathbb{R}^{4}$ with concentration scale $r_{c}$, there exists a unique finite energy solution $A$ to (1.10) in the time interval $\left[-r_{c}, r_{c}\right]$ in the temporal gauge $A_{0}=0$, depending continuously on the initial data. Furthermore, any other finite energy solution with the same data must be gauge equivalent to $A$.

(2) The same result holds for data in a ball $B_{R}$ and the solution in the corresponding domain of uniqueness $\mathcal{D}\left(C_{R}\right) \cap\left(\left[-r_{c}, r_{c}\right] \times \mathbb{R}^{4}\right)$.

We remark that this temporal gauge well-posedness result is in some sense a soft result, which is not accompanied by any dispersive type estimates. In expanded form, it asserts that regular data generates regular solutions on the $r_{c}$ time scale, and that the data to the solution map has a continuous extension to all finite energy data in the uniform energy norm. However, its proof is anything but straightforward, as it requires the full strength of the local well-posedness in the caloric gauge (cf. section 3.7).

Now we consider the continuation question. The next result asserts that temporal solutions can be continued until energy concentration (i.e., blowup) occurs. Thus, temporal solutions are also maximal solutions for the Yang-Mills equation.

\section{Theorem 4.7.}

(1) For each finite energy data $(a, e)$ in $\mathbb{R}^{4}$, let $\left(T_{\min }, T_{\max }\right)$ be the maximal time interval on which the temporal gauge solution exists. If $T_{\max }$ is finite, then we have

$$
\lim _{t \rightarrow T_{\max }} r_{c}(t)=0,
$$

and similarly for $T_{\min }$. Furthermore, there exits some $X \in \mathbb{R}^{4}$ so that energy concentrates in the backward light cone of $\left(T_{\max }, X\right)$ (respectively the forward light cone of $\left.\left(T_{\min }, X\right)\right)$.

(2) The same result holds for data in a ball $B_{R}$ and the solution in the corresponding domain of uniqueness $\mathcal{D}\left(B_{R}\right)$.

The main advantage of this theorem is that it allows us to work with solutions which do not admit a global caloric representation. The vanishing of $r_{c}(t)$ is a corollary of Theorem 4.6, while existence of an energy concentration point follows by a standard argument; see, e.g., [27, Lemma 8.1].

The temporal gauge is convenient in order to deal with causality, but not so much in terms of regularity, as it lacks good $S^{1}$-bounds. For this reason it is convenient to borrow the caloric gauge regularity:

Theorem 4.8. Let $A$ be a finite energy Yang-Mills solution in a cone section $C_{\left[t_{1}, t_{2}\right]}$ with energy concentration scale $r_{c}$. Then in a suitable gauge A satisfies the bound

$$
\|A\|_{L^{\infty}\left(\dot{H}^{1} \cap L^{4}\right)}+\left\|\partial_{t} A\right\|_{L^{\infty} L^{2}}+\left\|\partial^{j} A_{j}\right\|_{\ell^{1} \dot{H}^{\frac{1}{2}}}+\left\|A_{0}\right\|_{\ell^{1} \dot{H}^{\frac{3}{2}}}+\left\|\square A_{x}\right\|_{L^{2} \dot{H}^{-\frac{1}{2}}} \lesssim_{E, \frac{r_{c}}{t_{2}}} 1
$$


in the smaller cone $C_{\left[t_{1}, t_{2}\right]}^{4 r_{c}}$, where the radius has uniformly been decreased by $4 r_{c}$.

The proof of this theorem requires a good gluing technique for local connections with suitable regularity, which were used to prove Proposition 4.4 and Theorem 4.5 as well.

\section{To Bubble or NOT to BUbBle}

In this section we outline the proof of our two main results in Theorems 1.4 and 1.5. following our fourth and final article 33. This is a blow-up argument based on Morawetz-type monotonicity formulas, broadly following the outline of prior works on wave maps [41] and the Maxwell-Klein-Gordon equation [27]. However, new difficulties arise here, both at the conceptual level and at the technical level, due to the more nonlinear gauge features inherent in the Yang-Mills equation and to the nontrivial topological structure.

We start with a part common to both proofs, namely the energy-based criterion for soliton bubbling off, and then we consider the two results separately.

5.1. A bubble-off criterion. Our aim here is to describe the proof of the following result, which provides a bubbling-off criterion that applies equally for both the Threshold and the Dichotomy Theorems.

Theorem 5.1 (Bubbling Theorem).

(1) Let $A$ be a finite-energy Yang-Mills wave which blows up in finite time at $(T, X)$. Assume in addition that for some $\gamma<1$ we have

$$
\underset{t \nearrow T}{\limsup } \mathcal{E}_{C_{\gamma} \cap S_{t}}(A)>0, \quad C_{\gamma}=\{|x-X| \leq \gamma|t-T|\} .
$$

Then $A$ bubbles off a soliton at $(T, X)$, as described after Theorem 1.5 .

(2) Let $A$ be a finite-energy Yang-Mills wave which is global forward in time. Assume in addition that for some $\gamma<1$ we have

$$
\limsup _{t \nearrow \infty} \mathcal{E}_{C_{\gamma} \cap S_{t}}(A)>0, \quad C_{\gamma}=\{|x| \leq \gamma t\} .
$$

Then A bubbles off a soliton at infinity, as described after Theorem 1.5.

Here $\mathcal{E}_{C_{\gamma} \cap S_{t}}(A)$ denotes the standard energy of $A$ measured on the time $t$ section of the cone $C_{\gamma}$.

5.1.1. Beginning of the proof. We start with some notation and initial simplifications. In the finite time blow-up case, by translation and reflection we can assume that $(T, X)=(0,0)$, and that the blowup occurs in the forward light cone. We introduce the forward cone $C$, its lateral boundary $\partial C$ and the foliation $\left\{S_{t}\right\}_{t \in[0, \infty)}$ as

$$
C=\{(t, x): 0 \leq|x| \leq t\}, \quad \partial C=\{(t, x): 0 \leq|x|=t\}, \quad S_{t}=C \cap\left(\{t\} \times \mathbb{R}^{4}\right) .
$$

We introduce the energy flux $\mathcal{F}_{\left[t_{1}, t_{2}\right]}(A)$, defined as

$$
\mathcal{F}_{\left[t_{1}, t_{2}\right]}(A)=\mathcal{E}_{t_{2}}(A)-\mathcal{E}_{t_{1}}(A) .
$$

Assume, for simplicity, that $A$ is regular. Then in both scenarios, by the above energy flux relation, we can easily obtain a sequence $A^{(n)}$ of Yang-Mills waves, by rescaling the original $A$, which have the following properties:

(1) $A^{(n)}$ is defined on $C_{\left[\varepsilon_{n}, 1\right]}$ where $\varepsilon_{n} \rightarrow 0$; 
(2) (bounded energy in the cone) $\mathcal{E}_{S_{t}}\left(A^{(n)}\right) \leq E$ for every $t \in\left[\varepsilon_{n}, 1\right]$;

(3) (decaying flux on $\partial C) \mathcal{F}_{\left[\varepsilon_{n}, 1\right]}\left(A^{(n)}\right) \leq \varepsilon_{n}^{\frac{1}{2}} E$;

(4) (nontrivial time-like energy at $t=1) \mathcal{E}_{C_{\gamma} \cap S_{1}}\left(A^{(n)}\right) \geq E_{0}>0$.

5.1.2. A Morawetz identity. Here we describe the key monotonicity formula (or a Morawetz identity), from which we obtain both asymptotic stationarity and compactness for bubble extraction. The idea is to use the renormalized scaling vector field $X_{0}=\frac{1}{\sqrt{t^{2}-|x|^{2}}}\left(t \partial_{t}+x \cdot \partial_{x}\right)$ as a multiplier. Introducing

$$
{ }^{\left(X_{0}\right)} P_{\alpha}(A)=T_{\alpha \beta}(A) X_{0}^{\beta},
$$

where $T_{\alpha \beta}(A)$ is the Yang-Mills energy-momentum tensor, we have

$$
\operatorname{div}{ }^{\left(X_{0}\right)} P(A)=\frac{2}{\rho_{0}}\left|\iota_{X_{0}} F\right|^{2},
$$

where $\rho_{0}=\sqrt{t^{2}-|x|^{2}}$. Remarkably, the right-hand side is nonnegative!

To derive a monotonicity formula, we would like to integrate (5.3) on $C_{\left[t_{1}, t_{2}\right]}$ and apply the divergence theorem. However, this is not possible since the weight $\rho_{0}^{-1}$ blows up on $\partial C$. Instead we introduce a parameter $\varepsilon>0$ and consider $X_{\varepsilon}=$ $\frac{1}{\rho_{\varepsilon}}\left((t+\varepsilon) \partial_{t}+x \cdot \partial_{x}\right)$, where $\rho_{\varepsilon}=\sqrt{(t+\varepsilon)^{2}-|x|^{2}}$. Introducing the notation

$$
{ }^{\left(X_{\varepsilon}\right)} \mathcal{P}_{S_{t}}(A)=\int_{S_{t}}{ }^{\left(X_{0}\right)} P_{0}(A) d x
$$

we arrive at

$$
{ }^{\left(X_{\varepsilon}\right)} \mathcal{P}_{S_{t_{2}}}(A)+\int_{C_{\left[t_{1}, t_{2}\right]}} \frac{1}{\rho_{\epsilon}}\left|\iota_{X_{\epsilon}} F\right|^{2} d t d x={ }^{\left(X_{\varepsilon}\right)} \mathcal{P}_{S_{t_{1}}}+\int_{\partial C_{\left[t_{1}, t_{2}\right]}}{ }^{\left(X_{\epsilon}\right)} P_{\alpha}(A) L^{\alpha} d \text { Area }
$$

where $L=\partial_{t}+\frac{x}{|x|} \cdot \partial_{x}$. In the ideal case when the integral on $\partial C$ vanishes, (5.4) says that the quantity ${ }^{\left(X_{\varepsilon}\right)} \mathcal{P}_{S_{t}}$ is monotone in $t$.

To describe ${ }^{\left(X_{\varepsilon}\right)} \mathcal{P}_{S_{t}}$ in detail, we need more notation. Let $L=\partial_{t}+\frac{x}{|x|} \cdot \partial_{x}$, $\underline{L}=\partial_{t}-\frac{x}{|x|} \cdot \partial_{x}$, and let $\left\{e_{\mathfrak{a}}\right\}_{2,3,4}$ be orthonormal vectors which are orthogonal to $L, \underline{L}$. In terms of the null decomposition of $F$ defined as

$$
\alpha_{\mathfrak{a}}=F\left(L, e_{\mathfrak{a}}\right), \quad \underline{\alpha}_{\mathfrak{a}}=F\left(\underline{L}, e_{\mathfrak{a}}\right), \quad \varrho=\frac{1}{2} F(L, \underline{L}), \quad \sigma_{\mathfrak{a} \mathfrak{b}}=F\left(e_{\mathfrak{a}}, e_{\mathfrak{b}}\right),
$$

we have

$$
\begin{aligned}
{ }^{\left(X_{\varepsilon}\right)} \mathcal{P}_{S_{t}}(A)=\int_{S_{t}}( & \frac{1}{2}\left(\frac{t+r+\varepsilon}{t-r+\varepsilon}\right)^{1 / 2}\left(|\alpha|^{2}+|\varrho|^{2}+|\sigma|^{2}\right) \\
& \left.+\frac{1}{2}\left(\frac{t-r+\varepsilon}{t+r+\varepsilon}\right)^{1 / 2}\left(|\underline{\alpha}|^{2}+|\varrho|^{2}+|\sigma|^{2}\right)\right) d x .
\end{aligned}
$$

Finally, we discuss how (5.4) is applied to our setting. For the solution $A^{(n)}$ constructed above, the right-hand side of (5.4) can be bounded by $\lesssim E$ for $\varepsilon=\varepsilon_{n}$. We point out that the last term is bounded by the energy flux $\mathcal{F}_{\left[t_{1}, t_{2}\right]}(A)$. Thus,

$$
\sup _{t \in\left(\varepsilon_{n}, 1\right]}\left(X_{\varepsilon_{n}}\right) \mathcal{P}_{S_{t}}\left(A^{(n)}\right)+\iint_{C_{\left(\varepsilon_{n}, 1\right]}} \frac{1}{\rho_{\varepsilon_{n}}}\left|\iota_{X_{\varepsilon_{n}}} F^{(n)}\right|^{2} d t d x \lesssim E .
$$

Consider a time-like cone $C_{\gamma}=\{(t, x):|x| \leq \gamma t\}$ for any $0<\gamma<1$. Observe that $\rho_{\varepsilon} \simeq t$ and $X_{\varepsilon}$ is uniformly time-like in $C_{\gamma} \cap\{t \geq 2 \varepsilon\}$ (both statements are 
uniform as $\varepsilon \rightarrow 0$ but degenerate as $\gamma \rightarrow 1$ ). Thus, boundedness of the spacetime integral term in (5.6) implies logarithmic integrated decay of a uniformly time-like interior derivative of $F^{(n)}$ in $C_{\gamma}$; this decay is the source of asymptotic stationarity and compactness.

5.1.3. Propagating energy in time-like region. The monotonicity formula (5.4) suggests that the weighted energy ${ }^{\left(X_{0}\right)} \mathcal{P}_{S_{t}}\left(A^{(n)}\right)$ essentially increases toward the tip. Using a suitably localized version of the formula, we show that nontrivial energy persists in a time-like cone toward the tip:

$$
\mathcal{E}_{C_{\gamma} \cap S_{t}}\left(A^{(n)}\right) \geq E_{1} \quad \text { for } t \in\left[\varepsilon_{n}^{\frac{1}{2}}, \varepsilon_{n}^{\frac{1}{4}}\right],
$$

where we make $1-\gamma$ and $E_{1}$ smaller if necessary.

5.1.4. Final rescaling. After a pigeonhole argument and suitable rescalings, we obtain a sequence of caloric Yang-Mills waves on $\left[1, T_{n}\right] \times \mathbb{R}^{4}$ (where $T_{n} \rightarrow \infty$ ), which we still denote by $A^{n}$, with the following properties (final rescaled sequence):

(1) (bounded energy in the cone) $\mathcal{E}_{S_{t}}\left(A^{(n)}\right) \leq E \quad\left(t \in\left[1, T_{n}\right]\right)$;

(2) (small energy outside the cone) $\mathcal{E}_{\left(\{t\} \times \mathbb{R}^{4}\right) \backslash S_{t}}\left(A^{(n)}\right) \ll E \quad\left(t \in\left[1, T_{n}\right]\right)$;

(3) (nontrivial energy in a time-like region) $\mathcal{E}_{C_{\gamma} \cap S_{t}}\left(A^{(n)}\right) \geq E_{1} \quad\left(t \in\left[1, T_{n}\right]\right)$;

(4) (asymptotic self-similarity) for every compact subset $\tilde{C}$ of $C_{[1, \infty)}^{1}=\{(t, x) \in$ $C:|x-| t|| \geq 1\}$

$$
\iint_{\tilde{C}}\left|\iota_{X_{0}} F^{(n)}\right| d t d x \rightarrow 0 \quad \text { as } n \rightarrow \infty .
$$

5.1.5. Locating concentration scales. To extract a bubble, we now locate (locally) smallest concentration scales in $A^{(n)}$, which retain the decay (5.8). A combinatorial argument from [27] (based on [41]) establishes two possible scenarios (along a subsequence of $\left.A^{(n)}\right)$ :

(i) Time-like concentration. There exists $r>0$, a sequence of points $\left(t_{n}, x_{n}\right) \rightarrow$ $\left(t_{0}, x_{0}\right) \in \operatorname{Int}\left(C_{[1, \infty)}\right)$, and a sequence of scales $r_{n} \rightarrow 0$ such that

$$
\sup _{x \in B_{r}\left(x_{n}\right)} \mathcal{E}_{B_{r_{n}}(x)}\left(A^{(n)}\right)
$$

is uniformly small but nontrivial, yet

$$
\frac{1}{2 r_{n}} \int_{t_{n}-r_{n}}^{t_{n}+r_{n}} \int_{B_{r}\left(x_{n}\right)}\left|\iota_{V} F^{(n)}\right| d t d x \rightarrow 0 \quad \text { as } n \rightarrow \infty .
$$

where $V=X_{0}\left(t_{0}, x_{0}\right)$.

(ii) Self-similar concentration. For every set 14 of the form

$$
\tilde{C}=\left\{(t, x): 0 \leq|x|<t-\frac{1}{2}, 2^{j} \leq t<2^{j+1} \text { for some } j \in \mathbb{Z}\right\}
$$

there exists $r=r(\tilde{C})$ such that

$$
\sup _{x \in \tilde{C}} \mathcal{E}_{B_{r}(x)}\left(A^{(n)}\right)
$$

is uniformly small.

\footnotetext{
${ }^{14}$ In fact, any compact subset $\tilde{C}$ in the interior of $C_{[1, \infty)}^{1}$ would work.
} 
5.1.6. Local compactness result. In both scenarios, we would like to extract a limit modulo scalings, translations, and gauge transformations. To ensure that the limit is nontrivial and solves the hyperbolic Yang-Mills equation, we need a means to ensure compactness.

Theorem 5.2. Let $A^{(n)}$ be a sequence of finite-energy Yang-Mills connections in $[-2,2] \times \mathbb{R}^{4}$ which is locally uniformly bounded in the sense of (4.3). Let $Q=$ $[-1,1] \times B_{R}(0)$ and $2 Q=[-2,2] \times B_{2 R}(0)$. Assume that

$$
\lim _{n \rightarrow \infty}\left\|\iota_{X} F\right\|_{L^{2}(2 Q)}=0
$$

where $X$ is a smooth time-like vector field. Then on a subsequence we have

$$
A^{(n)} \rightarrow A \quad \text { in } H^{1}(Q),
$$

where $A$ is a solution to the Yang-Mills equation satisfying $\iota_{X} F=0$.

The idea of the proof is as follows. The $S^{1}$-bound implies uniform boundedness of $\left\|\square A^{(n)}\right\|_{L^{2} \dot{H}^{-\frac{1}{2}}}$. This in turn implies extra regularity away from the characteristic cone $\{|\tau|=|\xi|\}$ in frequency space, since $\square$ is elliptic there. Near the characteristic cone, we use the following equations for $A^{(n)}$ :

$$
\begin{aligned}
X^{\alpha} \partial_{\alpha} A_{j}^{(n)}-X^{\ell} \partial_{j} A_{\ell}^{(n)} & =-\left(\iota_{X} F^{(n)}\right)_{j}+\text { (smoother error) }, \\
X^{\ell} \partial_{0} A_{\ell}^{(n)} & =-\left(\iota_{X} F^{(n)}\right)_{0}+\text { (smoother error). }
\end{aligned}
$$

Although the system on the left-hand side is not elliptic, it is microlocally elliptic (of order 1) near the characteristic cone $\{|\tau|=|\xi|\}$ in frequency space. Inverting this system, and using the hypothesis $\iota_{X} F^{(n)} \rightarrow 0$ in $L^{2}(2 Q)$, we arrive at the decomposition

$$
A^{(n)}=A^{(n), \text { small }}+A^{(n), \text { smooth }}, \quad\left\|A^{(n), \text { small }}\right\|_{H^{1}(Q)} \rightarrow 0, \quad\left\|A^{(n), \text { smooth }}\right\|_{H^{1+\alpha}(Q)} \lesssim 1
$$

for some $\alpha>0$ (in fact, $\alpha=\frac{1}{2}$ ). Applying Rellich-Kondrachov to $A^{(n) \text {,smooth }}$, the theorem follows.

5.1.7. Extraction of limiting profiles. In order to apply Theorem 5.2 in section 5.1.5(i), we rescale and translate so that $B_{r_{n}}\left(x_{n}\right) \rightarrow B_{1}(0)$ and apply Theorem 4.8 to ensure the bound (4.3), uniformly on bounded sets. As a result, we extract a nontrivial finite energy stationary solution (i.e., a soliton).

In scenario (ii) of section 5.1.5. we apply a similar procedure to $B_{r}(0)$, where we rely on property (4) of section 5.1.4 of the final rescaled sequence for the decay hypothesis in Theorem 5.2. In this case, we extract a finite-energy self-similar solution on $C_{[1, \infty)}^{1}$, which is nontrivial thanks to property (3) of section 5.1.4.

5.1.8. Exclusion of the self-similar case. To conclude the bubble extraction argument, it remains to rule out scenario (ii) of section 5.1.5. i.e., to prove that every finite-energy self-similar solution is trivial.

By self-similarity, the solution restricted to the hyperbolic space $\mathbb{H}^{4}=\{(t, x)$ : $\left.t>0, t^{2}-|x|^{2}=1\right\}$ is a harmonic Yang-Mills connection. Recall that the harmonic Yang-Mills equation in dimension 4 is conformally invariant. Thus, by a stereographic projection, we obtain a harmonic Yang-Mills connection on $\mathbb{D}^{4}$, which we still denote by $A$. The finite-energy condition restricted to the hyperbolic space $\mathbb{H}^{4}$ essentially implies that, after a suitable gauge transformation, $A$ is smooth up to the boundary and $A\left\lceil_{\partial \mathbb{D}^{4}}\right.$ vanishes. By an elliptic unique continuation argument (applied to $F$ ), it follows that the solution is trivial. 
5.2. The threshold theorem. We first restate our Threshold Theorem in the caloric gauge. We will consider the global solvability question for the system (1.10) with initial data at time $t=0$,

$$
\left(A_{j}(0), \partial_{0} A_{j}(0)\right)=\left(A_{0 j}, B_{0 j}\right) \in T^{L^{2}} \mathcal{C} \subset \mathcal{H}:=\mathbf{H}\left(\mathbb{R}^{4}\right) \times L^{2}\left(\mathbb{R}^{4}\right) .
$$

Here the caloric gauge imposes a constraint on both $A_{0 j}$ and on $B_{0 j}$. As discussed before, the temporal components of the connection, namely $A_{0}$ and $\partial_{0} A_{0}$, are determined in an elliptic fashion in terms of $A_{x}$ and $\partial_{0} A_{x}$.

We will also consider higher regularity and (weak) Lipschitz dependence properties of the solutions, using the spaces

$$
\mathcal{H}^{\sigma}=\dot{\mathcal{H}}^{\sigma} \cap \mathcal{H}, \quad \dot{\mathcal{H}}^{\sigma}=\dot{H}^{\sigma}\left(\mathbb{R}^{4}\right) \times \dot{H}^{\sigma-1}\left(\mathbb{R}^{4}\right) .
$$

Now we can provide a more complete statement for our main result:

Theorem 5.3. The Yang-Mills system in the caloric gauge (1.10) is globally wellposed in $\mathcal{H}$ for all caloric initial data in $\mathcal{H}$ below the ground state energy, in the following sense:

(i) Regular data. If in addition the data set $\left(A_{0 j}, B_{0 j}\right)$ is more regular, $\left(A_{0 j}, B_{0 j}\right) \in \mathcal{H}^{N}$, then there exists a unique global regular caloric solution $\left(A_{j}, \partial_{0} A_{j}\right)$ $\in C\left(\mathbb{R}, \mathcal{H}^{N}\right)$, also with $\left(A_{0}, \partial_{0} A_{0}\right) \in C\left(\mathbb{R}, \mathcal{H}^{N}\right)$, which has Lipschitz dependence on the initial data locally in time in the $\mathcal{H}^{N}$ topology.

(ii) Rough data. The flow map admits an extension

$$
T^{L^{2}} \mathcal{C} \ni\left(A_{0 j}, B_{0 j}\right) \rightarrow\left(A_{\alpha}, \partial_{t} A_{\alpha}\right) \in C(\mathbb{R}, \mathcal{H})
$$

and which is continuous in the $\mathcal{H} \cap \dot{\mathcal{H}}^{s}$ topology for $s<1$ and close to 1 .

(iii) Weak Lipschitz dependence. The flow map is globally Lipschitz in the $\dot{\mathcal{H}}^{s}$ topology for $s<1$, close to 1 .

We remark that in effect the proof of the theorem provides a stronger statement, where the regularity of the solutions is described in terms of function spaces $S^{1}$, $S^{N}$ which incorporate, in particular, Strichartz norms, $X^{s, b}$ norms, and null frame spaces.

Implicit in Theorem 5.3 is also a scattering result; however, this is not so easy to state as it is a modified rather than linear scattering. In a weaker sense, one can think of scattering as simply the fact that the $S^{1}$-norm is finite.

In what follows we outline the proof, using Theorems [3.1, 3.2, and 5.1 as our starting point.

5.2.1. No bubbling. The first step here is to show that no bubbling can occur. Here, we closely follow the argument in 21 .

Indeed, assume by contradiction that a sequence $A^{(n)}$ of rescales and translates of $A$ converges locally in $H^{1}$ to a Lorentz transform of a nontrivial soliton $L_{v} Q$, which implies $L_{\text {loc }}^{2}$ convergence of curvature tensors $F^{(n)}$. So after taking a subsequence, for almost every $t$

$$
\mathcal{E}_{\{t\} \times B_{R}}\left(A^{(n)}\right)=\frac{1}{2} \int_{B_{R}}\left\langle F^{(n)}, F^{(n)}\right\rangle(t) \rightarrow \mathcal{E}_{\{t\} \times B_{R}}\left(L_{v} Q\right) \quad \text { for any } R>0,
$$

which in turn implies

$$
\mathcal{E}(Q) \leq \mathcal{E}(A)<2 E_{\mathrm{GS}}
$$

By Theorem 1.3, the only possibility for $Q$ is that $|\chi(Q)|=\mathcal{E}_{e}(Q)$. Moreover, since Lorentz transforms preserve the topological class, $\chi\left(L_{v}(Q)\right)=\chi(Q)$. 
By topological triviality of $A^{(n)}(t)$, we have $\chi\left(A^{(n)}(t)\right)=0$, and thus

$$
\int_{\mathbb{R}^{4} \backslash B_{R}(0)}-\left\langle F^{(n)} \wedge F^{(n)}\right\rangle(t)=-\int_{B_{R}(0)}-\left\langle F^{(n)} \wedge F^{(n)}\right\rangle(t) .
$$

By $L_{\text {loc }}^{2}$ convergence of $F^{(n)}$, the absolute value of the first term on the right-hand side can be made arbitrarily close to $|\chi(A)|=\mathcal{E}(Q)$ by taking $R$ very large. Using the Bogomoln'yi lower bound $|\langle F \wedge F\rangle| \leq \frac{1}{2}\left\langle F_{i j}, F^{i j}\right\rangle$ in $\mathbb{R}^{4} \backslash B_{R}$, it follows that

$$
\begin{aligned}
\mathcal{E}(A) & \geq \limsup _{n \rightarrow \infty}\left(\frac{1}{2} \int_{B_{R}}\left\langle F^{(n)}, F^{(n)}\right\rangle(t)+\left|\int_{\mathbb{R}^{4} \backslash B_{R}}\left\langle F^{(n)} \wedge F^{(n)}\right\rangle(t)\right|\right) \\
& \geq \mathcal{E}_{\{t\} \times B_{R}}\left(L_{v} Q\right)+\left|\int_{B_{R}}-\left\langle F\left[L_{v} Q\right] \wedge F\left[L_{v} Q\right]\right\rangle\right| \\
& \geq \mathcal{E}\left(L_{v} Q\right)+\mathcal{E}(Q)-o_{R \rightarrow \infty}(1) .
\end{aligned}
$$

Since $\mathcal{E}\left(L_{v} Q\right) \geq \mathcal{E}(Q) \geq E_{\mathrm{GS}}$, we reach a contradiction.

5.2.2. No blowup. Suppose a finite-time blowup occurs for a subthreshold caloric Yang-Mills wave. By translation invariance we can assume that the blowup happens at $(0,0)$, backward in time. By the small data result, we must have energy concentration in the forward light cone $C$ at $t=0$,

$$
\lim _{t \searrow 0} \mathcal{E}_{S_{t}}(A)>0 \text {. }
$$

On the other hand, as bubbling cannot occur, by Theorem 5.1 we must have

$$
\lim _{t \searrow 0} \mathcal{E}_{C_{\gamma} \cap S_{t}}(A)=0 \quad \forall \gamma<1 .
$$

To reach a contradiction, it would suffice to show that the energy dispersion decays near the tip of the cone,

$$
\lim _{t \searrow 0}\|F\|_{E D[0, t]}=0 .
$$

Then Theorem 3.2 would yield a bound for $\|A\|_{S^{1}[0, t]}$, which shows that the solution $A$ extends below $t=0$, and in particular the energy concentration (5.10) cannot occur.

One problem with this strategy is that we have no a priori knowledge about what happens outside the cone. To rectify this, we excise the outer part of the solution, so that we are left with a connection $\tilde{A}$ in a small time interval $\left[0, t_{0}\right]$, so that

(1) the two connections agree inside, $\tilde{A}=A$ in $C_{\left[0, t_{0}\right]}$;

(2) $\tilde{A}$ has small energy outside,

$$
\mathcal{E}_{\mathbb{R}^{4} \backslash C_{t}}(\tilde{A}) \leq \epsilon \ll 1, \quad t \in\left[0, t_{0}\right] .
$$

Here $\epsilon$ can be chosen arbitrarily small, and $t_{0}$ depends on $\epsilon$. This is achieved using Proposition 4.2 at a well-chosen time $t_{0}$, using the flux decay near the tip of the cone. By finite speed of propagation, note that the new and old solutions agree in $C$. In particular, the new solution also concentrates energy at $(0,0)$, and thus cannot be extended past 0 .

Taking into account (5.12) and (5.11) (the latter transfers from $A$ to $\tilde{A}$ ) for $\tilde{A}$, we see that the energy of $\tilde{A}$ must concentrate near the cone. Using the Morawetz estimate (5.6), we obtain as well a second energy bound inside the cone, namely

$$
\limsup _{t \rightarrow 0}{ }^{\left(X^{\gamma}\right)} \mathcal{P}_{S_{t}}[\tilde{A}] \lesssim \mathcal{E} 1, \quad \gamma<1
$$


This shows that in addition, only certain curvature components may be large near the cone.

Finally, we are in a position to show that $\tilde{A}$ is energy dispersed near the tip, and thus reach the desired contradiction by Theorem 3.2. This is done using the following result:

Proposition 5.4. Let $\left(A_{x}, \partial_{t} A_{x}\right)(t)$ be caloric Yang-Mills data with energy $\mathcal{E}<$ $2 E_{\mathrm{GS}}$. Then for each $\epsilon>0$, there exists $\gamma<1$ and $\delta>0$ so that the bounds

$$
\mathcal{E}_{C_{\gamma} \cap S_{t}(A)}(A)+\mathcal{E}_{\mathbb{R}^{4} \backslash S_{t}}(\tilde{A}) \leq \delta, \quad{ }^{\left(X^{\gamma}\right)} \mathcal{P}_{S_{t}}[\tilde{A}] \lesssim \mathcal{E} 1,
$$

imply

$$
\|F\|_{\mathrm{ED}[t]} \leq \epsilon .
$$

Indeed, by the huge weight near $\partial C$ in $\left(X_{\varepsilon_{n}}\right) \mathcal{P}_{S_{1}}(A)$ and smallness of energy elsewhere, all components of $F$ except for $\underline{\alpha}$ are small in $L^{2}$. To control $\underline{\alpha}$, it suffices to consider $F_{r \mathfrak{a}}=\alpha_{\mathfrak{a}}-\underline{\alpha}_{\mathfrak{a}}$ in the frame $\left(e_{t}=\partial_{t}, e_{r}=\partial_{r}, e_{2}, e_{3}, e_{4}\right)$. By the Yang-Mills equation and the Bianchi identity, they obey the following covariant div-curl system on spheres 15

$$
\begin{aligned}
\mathbf{D}_{\mathfrak{a}} F_{r \mathfrak{b}}-\mathbf{D}_{\mathfrak{b}} F_{r \mathfrak{a}} & =\mathbf{D}_{r} \sigma_{\mathfrak{a} \mathfrak{b}}, \\
\mathbf{D}^{\mathfrak{a}} F_{r \mathfrak{a}} & =\mathbf{D}^{\mathfrak{a}} \alpha_{\mathfrak{a}}+\mathbf{D}_{r} \varrho .
\end{aligned}
$$

The crucial observation is that the right-hand sides only involve components with small energy. In the commutative case (where $\mathbf{D}=\nabla$ ), this div-curl system can be easily inverted, and it follows that $\left\|\left|\nabla_{x}\right|^{-1} \not \nabla F_{r \mathfrak{a}}\right\|_{L^{2}} \ll E$, where $\not \nabla=$ $\left(\nabla_{e_{2}}, \nabla_{e_{3}}, \nabla_{e_{4}}\right)$ stands for the angular derivatives. By Bernstein, this is sufficient to rule out the null concentration scenario. A more involved argument is needed in the noncommutative case.

5.2.3. Scattering. The argument here is similar but simpler. Simply by translating the coordinate system, we can ensure that condition (5.12) holds for $t \in\left[t_{0}, \infty\right)$. Then the rest of the argument carries through unchanged.

5.3. The Dichotomy Theorem. Here we would like to apply the same argument as before. This time we are assuming, rather than proving, that bubbling does not happen. We can still truncate the solution $A$ outside to ensure that the bound (5.12) holds in the blow-up case or translate the coordinates to achieve the same outcome in the nonscattering case. The new difficulty is that we are no longer guaranteed that we can work in the caloric gauge, as the energy may be above the threshold.

However, it turns out that this is only a technical obstruction, as we can now prove a much stronger form of Proposition 5.4, namely:

Proposition 5.5. Let $\left(A_{x}, F_{0 x}\right)(t)$ be finite-energy Yang-Mills data with energy $\mathcal{E}$. Then for each $\epsilon>0$ there exists $\gamma<1$ and $\delta>0$ so that the bounds

$$
\mathcal{E}_{C_{\gamma} \cap S_{t}(A)}(A)+\mathcal{E}_{\mathbb{R}^{4} \backslash S_{t}}(\tilde{A}) \leq \delta, \quad{ }^{\left(X^{\gamma}\right)} \mathcal{P}_{S_{t}}[\tilde{A}] \lesssim{ }_{\mathcal{E}} 1,
$$

imply that $\left(A_{x}, F_{0 x}\right)(t)$ admits a caloric gauge representation so that in addition we have

$$
\|F\|_{\mathrm{ED}[t]} \leq \epsilon,
$$

\footnotetext{
${ }^{15}$ We remark that in our actual proof, we work with an analogous div-curl system on hyperplanes for technical simplicity.
} 
The difficulty here is to obtain the caloric gauge representation, without assuming any a priori bound on $\|A[t]\|_{\dot{H}^{1} \times L^{2}}$. This is done via multiple continuity arguments (in a manner resembling the proof of Uhlenbeck's lemma [53]), in several steps:

(i) Working in an annulus, use a continuity argument to show that one can obtain a local gauge in which $A$ is controlled in $\dot{H}^{1}$, with small $L^{4}$-norm.

(ii) Extend previous step to all of $\mathbb{R}^{4}$, by gluing small $\dot{H}^{1} \cap L^{4}$ connections obtained via Uhlenbeck's lemma inside the annulus and outside.

(iii) Use a second continuity argument to show that a corresponding caloric connection exists. Here the previous step is used to construct a path to 0 .

\section{ABOUT THE AUTHORS}

Sung-Jin Oh is CMC Research Professor at the Korea Institute of Advanced Study in Seoul. His main research interests are nonlinear hyperbolic/dispersive equations arising from geometry and mathematical physics.

Daniel Tataru is professor at University of California, Berkeley. His current research is in partial differential equations, including problems in nonlinear dispersive equations, water waves, integrable systems, and general relativity.

\section{REFERENCES}

[1] J. Bourgain, Global solutions of nonlinear Schrödinger equations, American Mathematical Society Colloquium Publications, vol. 46, American Mathematical Society, Providence, RI, 1999. MR 1691575

[2] D. M. Eardley and V. Moncrief, The global existence of Yang-Mills-Higgs fields in 4dimensional Minkowski space. I. Local existence and smoothness properties, Comm. Math. Phys. 83 (1982), no. 2, 171-191. MR649158

[3] D. M. Eardley and V. Moncrief, The global existence of Yang-Mills-Higgs fields in 4dimensional Minkowski space. II. Completion of proof, Comm. Math. Phys. 83 (1982), no. 2, 193-212. MR649159

[4] D. S. Freed and K. K. Uhlenbeck, Instantons and four-manifolds, Mathematical Sciences Research Institute Publications, vol. 1, Springer-Verlag, New York, 1984. MR757358

[5] C. Gavrus, Global well-posedness for the massive Maxwell-Klein-Gordon equation with small critical Sobolev data, preprint, (2016), arXiv:1610.03581.

[6] M. G. Grillakis, Regularity and asymptotic behaviour of the wave equation with a critical nonlinearity, Ann. of Math. (2) 132 (1990), no. 3, 485-509, DOI 10.2307/1971427. MR1078267

[7] M. G. Grillakis, Regularity for the wave equation with a critical nonlinearity, Comm. Pure Appl. Math. 45 (1992), no. 6, 749-774, DOI 10.1002/cpa.3160450604. MR.1162370

[8] M. Gursky, C. Kelleher, and J. Streets, A conformally invariant gap theorem in Yang-Mills theory, preprint (2017), arXiv:1708.01157

[9] F. Hélein, Harmonic maps, conservation laws and moving frames, 2nd ed., Cambridge Tracts in Mathematics, vol. 150, Cambridge University Press, Cambridge, 2002. Translated from the 1996 French original; With a foreword by James Eells. MR1913803

[10] C. E. Kenig and F. Merle, Global well-posedness, scattering and blow-up for the energycritical focusing non-linear wave equation, Acta Math. 201 (2008), no. 2, 147-212, DOI 10.1007/s11511-008-0031-6. MR2461508

[11] S. Klainerman and M. Machedon, Space-time estimates for null forms and the local existence theorem, Comm. Pure Appl. Math. 46 (1993), no. 9, 1221-1268, DOI 10.1002/cpa.3160460902. MR.1231427

[12] S. Klainerman and M. Machedon, On the Maxwell-Klein-Gordon equation with finite energy, Duke Math. J. 74 (1994), no. 1, 19-44, DOI 10.1215/S0012-7094-94-07402-4. MR.1271462

[13] S. Klainerman and M. Machedon, Finite energy solutions of the Yang-Mills equations in $\mathbb{R}^{3+1}$, Ann. of Math. (2) 142 (1995), no. 1, 39-119, doi 
[14] S. Klainerman and D. Tataru, On the optimal local regularity for Yang-Mills equations in $\mathbf{R}^{4+1}$, J. Amer. Math. Soc. 12 (1999), no. 1, 93-116, DOI 10.1090/S0894-0347-99-00282-9. MR.1626261

[15] J. Krieger, W. Schlag, and D. Tataru, Renormalization and blow up for the critical YangMills problem, Adv. Math. 221 (2009), no. 5, 1445-1521, DOI 10.1016/j.aim.2009.02.017. MR.2522426

[16] J. Krieger and J. Lührmann, Concentration compactness for the critical Maxwell-KleinGordon equation, Ann. PDE 1 (2015), no. 1, Art. 5, 208, DOI 10.1007/s40818-015-0004-y. MR3479062

[17] J. Krieger and W. Schlag, Concentration compactness for critical wave maps, EMS Monographs in Mathematics, European Mathematical Society (EMS), Zürich, 2012. MR2895939

[18] J. Krieger and J. Sterbenz, Global regularity for the Yang-Mills equations on high dimensional Minkowski space, Mem. Amer. Math. Soc. 223 (2013), no. 1047, vi+99, DOI 10.1090/S00659266-2012-00566-1. MR 3087010

[19] J. Krieger, J. Sterbenz, and D. Tataru, Global well-posedness for the Maxwell-Klein-Gordon equation in $4+1$ dimensions: small energy, Duke Math. J. 164 (2015), no. 6, 973-1040, DOI 10.1215/00127094-2885982. MR3336839

[20] J. Krieger and D. Tataru, Global well-posedness for the Yang-Mills equation in $4+1$ dimensions. Small energy, Ann. of Math. (2) 185 (2017), no. 3, 831-893, DOI 10.4007/annals.2017.185.3.3. MR3664812

[21] A. Lawrie and S.-J. Oh, A refined threshold theorem for $(1+2)$-dimensional wave maps into surfaces, Comm. Math. Phys. 342 (2016), no. 3, 989-999, DOI 10.1007/s00220-015-2513-7. MR3465437

[22] F. Lin and C. Wang, The analysis of harmonic maps and their heat flows, World Scientific Publishing Co. Pte. Ltd., Hackensack, NJ, 2008. MR2431658

[23] M. Machedon and J. Sterbenz, Almost optimal local well-posedness for the $(3+1)$-dimensional Maxwell-Klein-Gordon equations, J. Amer. Math. Soc. 17 (2004), no. 2, 297-359, DOI 10.1090/S0894-0347-03-00445-4. MR2051613

[24] V. Moncrief, Global existence of Maxwell-Klein-Gordon fields in $(2+1)$-dimensional spacetime, J. Math. Phys. 21 (1980), no. 8, 2291-2296, DOI 10.1063/1.524669. MR.579231

[25] S.-J. Oh, Gauge choice for the Yang-Mills equations using the Yang-Mills heat flow and local well-posedness in $H^{1}$, J. Hyperbolic Differ. Equ. 11 (2014), no. 1, 1-108, DOI 10.1142/S0219891614500015. MR3190112

[26] S.-J. Oh, Finite energy global well-posedness of the Yang-Mills equations on $\mathbb{R}^{1+3}$ : an approach using the Yang-Mills heat flow, Duke Math. J. 164 (2015), no. 9, 1669-1732, DOI 10.1215/00127094-3119953. MR.3357182

[27] S.-J. Oh and D. Tataru, Global well-posedness and scattering of the $(4+1)$-dimensional Maxwell-Klein-Gordon equation, Invent. Math. 205 (2016), no. 3, 781-877, arXiv: 1503.01562 doi

[28] S.-J. Oh and D. Tataru, Local well-posedness of the $(4+1)$-dimensional MaxwellKlein-Gordon equation at energy regularity, Ann. PDE 2 (2016), no. 1, Art. 2, 70, arXiv: 1503.01560 doi

[29] S.-J. Oh and D. Tataru, Energy dispersed solutions for the $(4+1)$-dimensional MaxwellKlein-Gordon equation, Amer. J. Math. 140 (2018), no. 1, 1-82, DOI 10.1353/ajm.2018.0000. MR.3749190

[30] S.-J. Oh and D. Tataru, The Yang-Mills heat flow and the caloric gauge, preprint (2017), arXiv: 1709.08599

[31] S.-J. Oh and D. Tataru, The hyperbolic Yang-Mills equation in the caloric gauge. Local well-posedness and control of energy dispersed solutions, preprint (2017), arXiv:1709.09332.

[32] S.-J. Oh and D.aniel Tataru, The hyperbolic Yang-Mills equation for connections in an arbitrary topological class, preprint (2017), arXiv:1709.08604

[33] S.-J. Oh and D. Tataru, The Threshold Conjecture for the energy critical hyperbolic YangMills equation, preprint (2017), arXiv:1709.08606

[34] P. Raphaël and I. Rodnianski, Stable blow up dynamics for the critical co-rotational wave maps and equivariant Yang-Mills problems, Publ. Math. Inst. Hautes Études Sci. 115 (2012), 1-122, DOI 10.1007/s10240-011-0037-z. MR2929728 
[35] I. Rodnianski and T. Tao, Global regularity for the Maxwell-Klein-Gordon equation with small critical Sobolev norm in high dimensions, Comm. Math. Phys. 251 (2004), no. 2, 377-426, DOI 10.1007/s00220-004-1152-1. MR2100060

[36] A. Schlatter, Long-time behaviour of the Yang-Mills flow in four dimensions, Ann. Global Anal. Geom. 15 (1997), no. 1, 1-25, DOI 10.1023/A:1006541305639. MR.1443269

[37] S. Selberg and A. Tesfahun, Null structure and local well-posedness in the energy class for the Yang-Mills equations in Lorenz gauge, J. Eur. Math. Soc. (JEMS) 18 (2016), no. 8, 1729-1752, DOI 10.4171/JEMS/627. MR3519539

[38] J. Shatah and M. Struwe, Regularity results for nonlinear wave equations, Ann. of Math. (2) 138 (1993), no. 3, 503-518, DOI 10.2307/2946554. MR.1247991

[39] L. M. Sibner, R. J. Sibner, and K. Uhlenbeck, Solutions to Yang-Mills equations that are not self-dual, Proc. Nat. Acad. Sci. U.S.A. 86 (1989), no. 22, 8610-8613, DOI 10.1073/pnas.86.22.8610. MR.1023811

[40] J. Sterbenz and D. Tataru, Energy dispersed large data wave maps in $2+1$ dimensions, Comm. Math. Phys. 298 (2010), no. 1, 139-230, DOI 10.1007/s00220-010-1061-4. MR2657817

[41] J. Sterbenz and D. Tataru, Regularity of wave-maps in dimension $2+1$, Comm. Math. Phys. 298 (2010), no. 1, 231-264, DOI 10.1007/s00220-010-1062-3. MR2657818

[42] M. Struwe, The Yang-Mills flow in four dimensions, Calc. Var. Partial Differential Equations 2 (1994), no. 2, 123-150, DOI 10.1007/BF01191339. MR.1385523

[43] M. Struwe, Variational methods, fourth ed., Ergebnisse der Mathematik und ihrer Grenzgebiete. 3. Folge. A Series of Modern Surveys in Mathematics [Results in Mathematics and Related Areas. 3rd Series. A Series of Modern Surveys in Mathematics], vol. 34, SpringerVerlag, Berlin, 2008, Applications to nonlinear partial differential equations and Hamiltonian systems.

[44] T. Tao, Global regularity of wave maps. II. Small energy in two dimensions, Comm. Math. Phys. 224 (2001), no. 2, 443-544, DOI 10.1007/PL00005588. MR.1869874

[45] T. Tao, Geometric renormalization of large energy wave maps, Journées "Équations aux Dérivées Partielles", École Polytech., Palaiseau, 2004, pp. Exp. No. XI, 32. MR2135366

[46] T. Tao, Global regularity of wave maps III. Large energy from $\mathbb{R}^{1+2}$ to hyperbolic spaces, preprint (2008), arXiv:0805.4666.

[47] T. Tao, Global regularity of wave maps IV. Absence of stationary or self-similar solutions in the energy class, preprint (2008), arXiv:0806.3592

[48] T. Tao, Global regularity of wave maps V. Large data local wellposedness and perturbation theory in the energy class, preprint (2008), arXiv:0808.0368

[49] T. Tao, Global regularity of wave maps VI. Abstract theory of minimal-energy blowup solutions, preprint (2009), arXiv:0906.2833

[50] T. Tao, Global regularity of wave maps VII. Control of delocalised or dispersed solutions, preprint (2009), arXiv:0908.0776.

[51] D. Tataru, On global existence and scattering for the wave maps equation, Amer. J. Math. 123 (2001), no. 1, 37-77. MR1827277

[52] D. Tataru, Rough solutions for the wave maps equation, Amer. J. Math. 127 (2005), no. 2, 293-377. MR2130618

[53] K. K. Uhlenbeck, Connections with $L^{p}$ bounds on curvature, Comm. Math. Phys. 83 (1982), no. 1, 31-42. MR648356

[54] K. K. Uhlenbeck, Removable singularities in Yang-Mills fields, Comm. Math. Phys. 83 (1982), no. 1, 11-29, http://projecteuclid.org/euclid.cmp/1103920742

Korea Institute for Advanced Study, Seoul, Korea 02455

Email address: sjoh@kias.re.kr

Department of Mathematics, University of California, Berkeley, Berkeley, CaliFORNIA, 94720

Email address: tataru@math.berkeley.edu 